\title{
Article
}

\section{A unified framework for the orbital structure of bars and triaxial ellipsoids}

\author{
Valluri, Monica, Shen, Juntai, Abbott, Caleb and Debattista, Victor P \\ Available at https://clok.uclan.ac.uk/14621/ \\ Valluri, Monica, Shen, Juntai, Abbott, Caleb and Debattista, Victor P orcid \\ iconORCID: 0000-0001-7902-0116 (2016) A unified framework for the orbital \\ structure of bars and triaxial ellipsoids. The Astrophysical Journal, 818 (2). p. \\ 141. ISSN 1538-4357
}

It is advisable to refer to the publisher's version if you intend to cite from the work. http://dx.doi.org/10.3847/0004-637X/818/2/141

For more information about UCLan's research in this area go to http://www.uclan.ac.uk/researchgroups/ and search for < name of research Group>.

For information about Research generally at UCLan please go to http://www.uclan.ac.uk/research/

All outputs in CLoK are protected by Intellectual Property Rights law, including Copyright law. Copyright, IPR and Moral Rights for the works on this site are retained by the individual authors and/or other copyright owners. Terms and conditions for use of this material are defined in the policies page.

\section{CLoK}

Central Lancashire online Knowledge www.clok.uclan.ac.uk

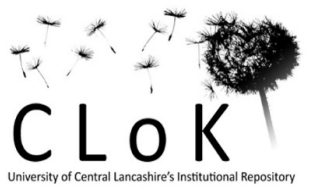




\title{
A UNIFIED FRAMEWORK FOR THE ORBITAL STRUCTURE OF BARS AND TRIAXIAL ELLIPSOIDS
}

\author{
Monica Valluri ${ }^{1}$, Juntai Shen $^{2}$, Caleb $^{\text {Abbott }}{ }^{1}$, and Victor P. Debattista ${ }^{3}$ \\ ${ }^{1}$ Department of Astronomy, University of Michigan, Ann Arbor, MI 48109, USA; mvalluri@umich.edu, calebga@umich.edu \\ 2 Key Laboratory for Research in Galaxies and Cosmology, Shanghai Astronomical Observatory, Chinese Academy of Sciences, \\ 80 Nandan Road, Shanghai 200030, China; jshen@shao.ac.cn \\ 3 Jeremiah Horrocks Institute, University of Central Lancashire, Preston, PR1 2HE, UK; vpdebattista@uclan.ac.uk \\ Received 2015 August 21; accepted 2016 January 8; published 2016 February 17
}

\begin{abstract}
We examine a large random sample of orbits in two self-consistent simulations of $N$-body bars. Orbits in these bars are classified both visually and with a new automated orbit classification method based on frequency analysis. The well-known prograde $\mathrm{x} 1$ orbit family originates from the same parent orbit as the box orbits in stationary and rotating triaxial ellipsoids. However, only a small fraction of bar orbits $(\sim 4 \%)$ have predominately prograde motion like their periodic parent orbit. Most bar orbits arising from the $\mathrm{x} 1$ orbit have little net angular momentum in the bar frame, making them equivalent to box orbits in rotating triaxial potentials. In these simulations a small fraction of bar orbits $(\sim 7 \%)$ are long-axis tubes that behave exactly like those in triaxial ellipsoids: they are tipped about the intermediate axis owing to the Coriolis force, with the sense of tipping determined by the sign of their angular momentum about the long axis. No orbits parented by prograde periodic $\mathrm{x} 2$ orbits are found in the pure bar model, but a tiny population $(\sim 2 \%)$ of short-axis tube orbits parented by retrograde $\mathrm{x} 4$ orbits are found. When a central point mass representing a supermassive black hole (SMBH) is grown adiabatically at the center of the bar, those orbits that lie in the immediate vicinity of the SMBH are transformed into precessing Keplerian orbits that belong to the same major families (short-axis tubes, long-axis tubes and boxes) occupying the bar at larger radii. During the growth of an SMBH, the inflow of mass and outward transport of angular momentum transform some $\mathrm{x} 1$ and long-axis tube orbits into prograde short-axis tubes. This study has important implications for future attempts to constrain the masses of SMBHs in barred galaxies using orbit-based methods like the Schwarzschild orbit superposition scheme and for understanding the observed features in barred galaxies.
\end{abstract}

Key words: chaos - galaxies: kinematics and dynamics - galaxies: nuclei - galaxies: spiral - galaxies: structure methods: numerical

\section{INTRODUCTION}

Historically, the study of orbits in potentials has focused on periodic orbits. In systems like disk galaxies small perturbations to closed periodic orbits (e.g., the epicyclic and vertical perturbations of circular orbits) provided a good analytic description of most orbits. Self-consistent distribution functions are thought to be "parented" by stable periodic orbits (Arnold 1978). Early works (e.g., Contopoulos \& Papayannopoulos 1980) identified and characterized the stability properties of the periodic orbit families in rapidly rotating bars. The most important periodic families in two-dimensional bars were identified as the prograde $\mathrm{x} 1$ family, which is elongated along the major axis of the bar, and the prograde stable $\mathrm{x} 2$ and unstable $\mathrm{x} 3$ families, which are elongated perpendicular to the bar (primarily found at small radii). The retrograde $\mathrm{x} 4$ (stable) orbit family is also elongated perpendicular to the bar at small radii, but becomes rounder as it extends to larger radii (for detailed description of orbit families and how they are identified see Contopoulos \& Grosbol 1989; Sellwood \& Wilkinson 1993; Binney \& Tremaine 2008; Sellwood 2014b). In the frame of reference rotating with the bar, all of these families are characterized by a 1:2 resonance between the tangential oscillation frequency $\left(\Omega_{\phi}\right)$ and the radial or epicyclic frequency $\left(\Omega_{R}\right)$. Indeed, studies of orbits in 2D N-body bars largely confirmed the picture arising from the study of periodic orbits and showed that many regular orbits elongated along the bar were parented by $\mathrm{x} 1$ orbits, a small fraction were parented by retrograde $\mathrm{x} 4$ orbits (Sparke \& Sellwood 1987), and none were parented by prograde $\mathrm{x} 2$ orbits. The realization that bars can also undergo buckling instabilities (Combes \& Sanders 1981; Raha et al. 1991), which makes them develop substantial vertical thickness and peanut-shaped morphologies, led to the study of periodic orbits in three-dimensional bars (Pfenniger 1984; Martinet \& de Zeeuw 1988; Pfenniger \& Friedli 1991; Skokos et al. 2002a, 2002b). It was shown that the appearance of specific morphological features in images of bars, such as the X-shape and peanut features seen in edge-on bars and the boxy/rectangular isophotes and "ansae" of face-on bars, could be explained by orbits trapped around specific periodic orbit families (Patsis et al. 2002, 2003, 2010). The introduction of a third dimension did not drastically change the picture of the nature of periodic orbits, and it was found that 3D bars are composed primarily of vertical bifurcations (resonances) of the $\mathrm{x} 1$ family and a few additional families (e.g., Pfenniger \& Friedli 1991; Skokos et al. 2002a, 2002b). Most studies of periodic orbits in analytic potentials consider prograde $\mathrm{x} 2$ orbits (but not retrograde $\mathrm{x} 4$ orbits) to be another fundamental building block of bars (Skokos et al. 2002a; Binney \& Tremaine 2008). In our study we do not find any orbits parented by the periodic prograde $\mathrm{x} 2$ orbit in our initial bar model, but we do find orbits that are parented by the periodic retrograde $\mathrm{x} 4$ orbit - a result that is consistent with previous studies (Sparke \& Sellwood 1987; Pfenniger \& Friedli 1991; Voglis et al. 2007).

In contrast with the study of bars, which has largely focused on planar periodic orbits and their vertical bifurcations, the orbits in triaxial ellipsoids are considered to belong to four 
regular families that occupy three dimensions: (a) short-axis tubes, ${ }^{4}$ (b) inner long-axis tubes, (c) outer long-axis tubes, and (d) box orbits and resonant boxlets (de Zeeuw 1985a; Statler 1987; Miralda-Escude \& Schwarzschild 1989). These families are primarily distinguished by their net angular momentum: short $(z)$ axis tubes have nonzero angular momentum about the short axis $\left(\left\langle J_{z}\right\rangle \neq 0\right)$; both families of long-axis tubes have a net angular momentum about the long $(x)$ axis $\left(\left\langle J_{x}\right\rangle \neq 0\right)$; boxes and boxlets have no net angular momentum about any axis. In addition, in a realistic triaxial potential, a significant fraction of orbits may be chaotic (e.g., Schwarzschild 1993; Merritt \& Fridman 1996).

The earliest studies of orbits in a triaxial system also examined orbital structure under the premise that the major families arise from perturbations of stable periodic orbits. For example, when discussing the numerical distributions derived by Schwarzschild (1979), de Zeeuw (1985b) states that "phasespace is well ordered and [...] most orbits belong to one of a few major families, each connected with a simple periodic orbit." De Zeeuw (1985a) showed that equations of motion in a triaxial ellipsoid were separable in ellipsoidal coordinates and that the major orbit families (the boxes and three families of tubes) had well-defined shapes and were characterized by welldefined relationships between their integrals of motion.

In this framework box orbits arise from perturbations (in two perpendicular directions $y$ and $z$ ) to the $x$-axial orbit (i.e., they are Lissajous curves in three dimensions). Since box orbits are composed of orthogonal perturbations of the $x$-axial orbit, they have no net angular momentum about any axis of symmetry. In contrast, short-axis tubes arise from perturbations (in the radial and $z$ direction) to a closed (periodic) elliptical orbit that circulates about the $z$-axis in the $x-y$ plane. Consequently, short-axis tubes have a net angular momentum about the $z$-axis. Likewise, long-axis tubes arise from perturbations to a closed (periodic) elliptical orbit in the $y-z$ plane and have a net angular momentum about the $x$-axis. Intermediate-axis tube orbits do not exist since elliptical orbits that circulate in the $x-z$ plane perpendicular to the intermediate axis are unstable (Heiligman \& Schwarzschild 1979; Adams et al. 2007).

When a triaxial potential is subjected to rotation of the figure about the $z$-axis, the $x$-axial orbit no longer oscillates back and forth along the long axis of the figure. Instead, in the frame that is co-rotating with the potential, such a particle experiences a Coriolis force whose sign changes each time the orbit reverses direction. Consequently, the particle follows an elliptical trajectory (Schwarzschild 1982; de Zeeuw \& Merritt 1983; Martinet \& de Zeeuw 1988) in a prograde sense about the $z$-axis.

Heisler et al. (1982) showed that when subjected to figure rotation (about the short axis), the clockwise and anticlockwise 1:1 periodic loop orbits circulating about the long axis are tipped about the intermediate axis by the Coriolis force, giving them additional retrograde angular momentum about the short axis. These orbits are referred to as "anomalous," and stable and unstable versions exist. The direction in which these orbits are tipped about the $y$-axis depends on the sign of $J_{x}$. At large energies these orbits become complex unstable (Martinet \& de Zeeuw 1988; Patsis \& Zachilas 1990). The anomalous orbits are also connected with the $\mathrm{x} 4$ family (Pfenniger \& Friedli

\footnotetext{
4 Hereafter we will use a coordinate system in which the long axis of the potential is aligned with the $x$-axis, the short axis of the potential is aligned with the $z$-axis, and the intermediate axis is aligned with the $y$-axis.
}

1991). Since the loop orbits circulating around the long axis are the parents of the long-axis tube family in a stationary triaxial potential, it follows that the stable anomalous orbits parent the long-axis tube families in a rotating triaxial potential. Consequently, long-axis tube orbits are also tipped about the intermediate axis in a rotating system (Deibel et al. 2011).

Finally, while short-axis tubes remain stable under figure rotation, the phase space occupied by prograde short-axis tubes decreases dramatically with increasing pattern speed, and they are increasingly replaced by retrograde short-axis tubes (Martinet \& de Zeeuw 1988; Deibel et al. 2011).

Since bars form via secular instabilities from rapidly rotating disks while stationary or slowly tumbling triaxial ellipsoids are thought to form primarily from collisionless mergers, in the current literature these systems are usually considered to be fundamentally different from each other (Binney \& Tremaine 2008). However, early studies of rotating triaxial figures (e.g., Heisler et al. 1982; Schwarzschild 1982; de Zeeuw \& Merritt 1983; Martinet \& de Zeeuw 1988) predicted that the behaviors of orbits in rotating triaxial systems and bars were fundamentally similar.

In this paper we examine two high-resolution $N$-body simulations of bars with and without a point mass representing a supermassive black hole $(\mathrm{SMBH})$ and show that all the families of orbits in triaxial ellipsoids are present in selfconsistent distribution functions of $N$-body bars as previously predicted. Unlike studies that analyze the periodic orbits in an analytic potential, our goal is to examine the orbits that compose the actual distribution functions of $N$-body bars with and without SMBHs. Our main goals are (a) to develop an automated orbital classification method for bar orbits and (b) to use it to understand how the orbit populations are modified by the growth of an SMBH of realistic mass fraction.

In Section 2 we describe the $N$-body simulations of selfconsistent bars and the prolate triaxial Dehnen model used to illustrate the similarity with orbits in $N$-body bars. In Section 3 we characterize the main orbit families present in our two $\mathrm{N}$ body bars and compare them with orbits integrated in stationary and rotating triaxial Dehnen models. In Section 4 we compare the orbit populations in the pure bar with the populations in the bar after the growth of a central point mass representing an SMBH. We also characterize the phase-space distribution of the bar models using both surfaces-of-section (SoSs) and frequency maps and show how the orbit populations vary with orbital apocenter radius. In Section 5 we discuss the implications of this work and summarize our results. In Appendix A we briefly describe the orbital frequency analysis method, and in Appendix B we describe our new automatic classification scheme for orbits in $N$-body bars.

\section{METHODS}

\subsection{Simulations}

The $N$-body disk models studied in this paper are almost identical to those presented in Shen \& Sellwood (2004, hereafter SS04) and were previously analyzed by Brown et al. (2013), who examined the effects of the growth of SMBHs on the measurement of the observable velocity dispersion within the effective radius $\left(\sigma_{e}\right)$ and the resultant consequences for the scatter and slope of the well-known scaling relationship between SMBH mass $\left(M_{\mathrm{BH}}\right)$ and stellar velocity dispersion $(\sigma)$ : the " $M_{\mathrm{BH}}-\sigma$ relationship." Below we give a brief 
description of the simulations and refer the reader to the above papers for a more detailed discussion of the setup and simulation process.

In this paper we restrict our orbital analysis to a single set of initial conditions composed of a Kuzmin disk embedded in a static logarithmic halo (for details see SS04). Following standard practice, the units used in the simulations are $G=M_{d}=R_{d}=1$, where $G$ is Newton's gravitational constant, $M_{d}$ is the mass of the disk, and $R_{d}$ is the initial disk scale radius. By dimensional analysis the unit of time is $t_{\text {dyn }}=\left(R_{d}^{3} / G M_{d}\right)^{1 / 2}$. In this paper all figures are presented in these units. Physically relevant scalings can be obtained by choosing observationally motivated values for $M_{d}$ and $R_{d}$. For example, $M_{d}=5 \times 10^{10} M_{\odot}$ and $R_{d}=1 \mathrm{kpc}$ would yield a unit of time $t_{\mathrm{dyn}} \sim 2.1 \mathrm{Myr}$. In these units the semimajor axis length of the bar is about 3-4 kpc in length and the disk has a total radius of about $25 \mathrm{kpc}$, making it similar to the Milky Way.

The initial disk distribution function consisted of $2.8 \times 10^{6}$ disk particles set up with a Toomre $Q$ parameter $\simeq 1.5$, a condition that ensures that it is unstable to bar formation (Athanassoula \& Sellwood 1986). The initial conditions were evolved using a three-dimensional, cylindrical, polar-gridbased $N$-body code described in Sellwood \& Valluri (1997) and Sellwood (2014a).

The disk formed a bar that subsequently experienced vertical thickening via the buckling instability (Toomre 1966, p. 111; Raha et al. 1991; Sellwood \& Wilkinson 1993) and developed the peanut-shaped bulge that is characteristic of this instability. After the disk reached a nearly steady state (simulation time $t_{1}=700$ ), a central point mass ${ }^{5}$ representing an SMBH of mass $0.2 \% M_{d}$ was grown adiabatically (for details see Brown et al. 2013). At $t_{2}=1200$ the transients due to the changing central point mass $(\mathrm{SMBH})$ potential had dissipated and the simulation was frozen, and orbits were examined and compared with orbits from the frozen potential at $t_{1}$.

Hereafter we refer to the frozen snapshots at $t_{1}$ and $t_{2}$ as Model A and Model B, respectively. The bar pattern speed at $t_{1}$ and $t_{2}$ was computed from several successive time steps before and after each snapshot. The galaxy potential for each snapshot is derived from the full $\mathrm{N}$-body distribution and is not described by an analytic form. Since our objective is to understand the actual orbits that populate the $\mathrm{N}$-body distribution function, we randomly select 10,000 particles (from the total of $\sim 2.8$ million) uniformly sampling the entire distribution function. The instantaneous positions and velocities of the selected particles (transformed to the rotating frame of the bar) were used to advance each of the 10,000 particles individually while keeping the rest of the particles fixed, by using a modification of the $N$-body code used to run the simulations. Orbit integration was carried out as described in footnote 10. The orbits were integrated in Cartesian coordinates ${ }^{6}$ in the rotating frame using the accelerations derived from the frozen potential of that snapshot and adding the appropriate Coriolis and centrifugal pseudoforces determined by the bar pattern speed (see Equations (2)-(4)). Each orbit was integrated for 1000 time units (corresponding to $\sim 2.1 \times 10^{9}$ yr for the units adopted above) and sampled at 20,000 equally spaced time

\footnotetext{
5 With softening parameter $\epsilon_{\mathrm{cmc}}=0.001 R_{d}$.

6 The Cartesian coordinate system was oriented with the major axis of the bar aligned with the $x$-axis, and with the angular momentum of the disk aligned with the $z$-axis, and the intermediate axis of the bar along the $y$-axis.
}

intervals. Since orbits at different radii have different orbital periods, this corresponds to hundreds of orbital periods for the innermost particles but to just tens of orbital periods for orbits in the outskirts of the disk.

Since the 10,000 particles for which orbits were integrated were selected at random from the entire distribution function, disk particles were included. The bar length in $N$-body simulations is generally estimated to be the radius at which the strength of the bar mode $A_{2}$ drops to some fraction (e.g., $1 / 2$ ) of its maximum value (Athanassoula \& Misiriotis 2002). By this criterion the bar length in this simulation is estimated to be about 3 (in program length units). However, visual classification of all 10,000 orbits in Model A showed that a significant fraction of bar-like orbits continue to exist all the way out to $\sim 4$ units in length (see the Appendix).

\subsection{The Triaxial Dehnen Potential}

We compare orbits from the bar snapshots with orbits in stationary and rotating triaxial potentials that are generalizations of the spherical "Dehnen models" (Dehnen 1993; Tremaine et al. 1994). These models have density profiles that provide good fits to luminosity profiles of elliptical galaxies and the bulges of spiral galaxies. The density distribution in these models is stratified on concentric ellipsoids with principle axes aligned with Cartesian coordinates $x, y, z$ and with the semimajor, semi-intermediate, and semiminor axis lengths $a, b, c$, respectively. The parameter $\gamma$ determines the logarithmic slope of the central density cusp and ranges observationally from $\gamma=0.1-1$ in bright galaxies with shallow cusps to $\gamma=2$ in fainter galaxies with steep cusps (Gebhardt et al. 1996; Lauer et al. 2007). We restrict ourselves to models with $\gamma=0.1$ since more cuspy models produce many resonant boxlet orbit families (Miralda-Escude \& Schwarzschild 1989; Merritt \& Valluri 1999) that are not found in our $N$-body bars. Equations describing the potential and the accelerations for this model are given in Merritt \& Fridman (1996) and Deibel et al. (2011).

In a rotating frame, the Jacobi integral $\left(E_{\mathrm{J}}\right)$ is a conserved quantity (equivalent to energy in a stationary frame):

$$
E_{\mathrm{J}}=\frac{1}{2}|\dot{\boldsymbol{x}}|^{2}+\Phi-\frac{1}{2}\left|\Omega_{p} \times \boldsymbol{x}\right|^{2}
$$

where $\boldsymbol{x}$ and $\dot{\boldsymbol{x}}$ are three-dimensional spatial and velocity vectors, respectively. When the rotation is about the short axis $(z)$, the equations of motion in Cartesian coordinates are given by

$$
\begin{gathered}
\ddot{x}=-\frac{\partial \Phi}{\partial x}+2 \Omega_{p} \dot{y}+\Omega_{p}^{2} x, \\
\ddot{y}=-\frac{\partial \Phi}{\partial y}-2 \Omega_{p} \dot{x}+\Omega_{p}^{2} y, \\
\ddot{z}=-\frac{\partial \Phi}{\partial z},
\end{gathered}
$$

where $2 \Omega_{p} \dot{y}$ and $-2 \Omega_{p} \dot{x}$ are components of the Coriolis force and $\Omega_{p}^{2} x$ and $\Omega_{p}^{2} y$ are components of the centrifugal force. Following standard practice, we adopt the right-handed rule where motion anticlockwise about the $z$-axis (as viewed from positive $z$ ) has positive angular momentum, while clockwise motion about the $z$-axis has negative angular momentum. In this terminology the direction of the pattern rotation of the 
simulated bars and the direction in which the triaxial Dehnen model is rotating when viewed from an inertial frame are both positive (anticlockwise). Similarly, when we discuss shortaxis (long-axis) tubes, anticlockwise motion about the $z$-axis ( $x$-axis) corresponds to positive angular momentum $J_{z}\left(J_{x}\right)$.

Deibel et al. (2011) studied orbits in triaxial Dehnen models with a variety of shapes subjected to a range of pattern speeds. Here we restrict our comparison to orbits in a prolate triaxial potential with axis ratios $c / a=0.4, b / a=0.48$, and hence triaxiality parameter $T=\left(a^{2}-b^{2}\right) /\left(a^{2}-c^{2}\right)=0.916$ and a weak cusp $(\gamma=0.1)$. This shape is quite close to that of the bars in our $N$-body simulations. As in the case of the $N$-body simulation, we adopt a set of units where the total mass of the model $M$, the semimajor axis scale length $a$, and the gravitational constant $G$ are set to unity.

Following standard practice, the patten speed used to describe the tumbling of the triaxial figure is defined in terms of the "co-rotation radius," hereafter $R_{\mathrm{CR}}$. In a nearly axisymmetric potential the co-rotation radius is the radius at which the azimuthal frequency of a closed (almost circular) orbit in the equatorial $(x-y)$ plane of the potential is the same as the pattern frequency (generally called "pattern speed") $\Omega_{p}$. The pattern speeds of bars have been measured by applying the Tremaine-Weinberg method (Tremaine \& Weinberg 1984; Meidt et al. 2008) to stellar kinematical velocity fields (as well as $\mathrm{H} \alpha$ and $\mathrm{HI}_{\mathrm{I}}$. These measurements find that the ratio of the co-rotation radius to the length of the bar is $R_{\mathrm{CR}} / R_{\mathrm{bar}}=[1$, 1.4] for a bar of length $R_{\text {bar }}$ (e.g., Merrifield \& Kuijken 1995; Debattista et al. 2002; Debattista \& Williams 2004; Aguerri et al. 2003; Corsini 2011; Aguerri et al. 2015). For the bar in the simulations described in the previous section, $R_{\mathrm{CR}} / R_{\mathrm{bar}} \sim 1$. For the orbits in the triaxial Dehnen model the pattern speed was chosen so that the co-rotation radius was at roughly the same radial distance from the center as it is in the case of the bar simulations (i.e., at $\sim 3$ units or one bar length). Note that the triaxial ellipsoidal surface with major-axis length $\sim 3$ in the Dehnen model encloses only $1 / 2$ of the total mass of the model. To ensure a fair comparison, the orbits from the stationary and rotating triaxial Dehnen model were selected to have similar radial extents to the bar orbits that they are compared with.

\subsection{Orbit Classification}

Orbits from the $N$-body bars were classified both visually and using our new automated orbit classification algorithm. All 10,000 orbits in Models A and B were visually classified by C.A. In addition, the innermost $\sim 4000$ particles of Model A were visually classified by M.V. Visual classification was based on $x-y, x-z, y-z$ projections and plots of angular momenta as a function of time $\left(J_{x}(t), J_{z}(t)\right)$ (examples of such plots are given in Figures 1, 4, 5, 6). We adopted classifications based on the full orbit (integration time $t=1000$ ), which is $\sim 200$ orbital periods for the innermost bar orbits but only $\sim 30$ orbital periods for the outermost disk particles. Although all orbits in an $\mathrm{N}$-body system are mildly chaotic (e.g., Miller 1964; Hemsendorf \& Merritt 2002), for the purpose of testing our automated classification scheme, we also attempt to visually classify chaotic orbits, although this is not possible to do in a robust manner. An orbit was visually classified as chaotic only if it could not be easily identified with a major orbit family (box, short-axis tube, long-axis tube), or if it showed signs of changing from one family to another during the integration time (indicating that it is sticky chaotic). For the $\sim 4000$ orbits that were visually classified by two of us (C.A. and M.V.) we found that fewer than $3 \%$ of orbits were classified differently. Where classifications disagreed, the orbits were generally transitional orbits-probably lying close to the separatrix between two families.

In Model A visual classification resulted in fewer chaotic orbits than were found by the automatic classification method (which relies on orbital frequency drift; see Appendix A); however, both methods yielded similar numbers of chaotic orbits in Model B. It is difficult to visually identify weakly chaotic (sticky chaotic) orbits since these typically lie at the edge of a resonant island or between two resonances and can appear regular for long times.

In Section 4.2 we show frequency maps with several minor resonant families. In frequency maps resonant orbits appear clustered along thin lines that satisfy a resonance condition $l \Omega_{x}+m \Omega_{y}+n \Omega_{z}=0$ (where $l, m, n$ are small integers). The signs of the integers indicate the phase relationship between the frequency components. The automatic classification method easily identifies resonant orbits, but these are visual identified only if they are quite close to the resonant parent orbit. Weakly resonant orbits are visually classified as boxes.

Voglis et al. (2007) used the orbital fundamental frequencies in cylindrical polar coordinates $\left(\Omega_{R}, \Omega_{\phi}, \Omega_{z}\right)$ to classify orbits in an $N$-body bar. In Appendix B we show that a much clearer separation of orbit families is obtained with fundamental frequencies computed in Cartesian coordinates. Our new automatic bar orbit classification scheme is described in detail in Appendix B, and a detailed comparison between the automatic and visual classification schemes is deferred to Section 4.1.

\section{ORBITAL BUILDING BLOCKS OF BARS}

\section{1. xl Orbits and Box Orbits}

The family of orbits parented by the periodic $\mathrm{x} 1$ orbit is considered to be the main bar-supporting orbit family (see Binney \& Tremaine 2008, hereafter BT08). As mentioned previously, a particle on such an orbit makes two excursions in radius during each complete circuit in the azimuthal angle $\phi$ (e.g., Contopoulos \& Papayannopoulos 1980), and therefore the radial oscillation frequency and tangential frequency are resonant $\Omega_{R}: \Omega_{\phi}=2: 1$. Since its apocenter radius increases with increasing Jacobi integral $E_{\mathrm{J}}$, at large $E_{\mathrm{J}}, \mathrm{x} 1$ orbits develop loops at their extremities. A particle on an $\mathrm{x} 1$ orbit travels in a prograde sense about the center of the galaxy (i.e., in the same sense that the bar pattern is rotating) except in the loops where the motion is retrograde relative to the figure.

In triaxial ellipsoids the main orbit family responsible for providing the high density along the long axis is the threedimensional box orbit family (BT08). In a stationary potential boxes have no net angular momentum about any axis. However, in a rotating frame Coriolis forces result in "envelope doubling," which imparts a small net angular momentum to the parent $x$-axis orbit, as well as box orbits (Schwarzschild 1982; de Zeeuw \& Merritt 1983). The "x1 orbit" is therefore also the parent of the quasi-periodic box orbit family in a rotating triaxial potential (Martinet \& de Zeeuw 1988). In this section we select orbits from our self-consistent $N$-body bar simulations to illustrate that the vast majority of orbits in these 

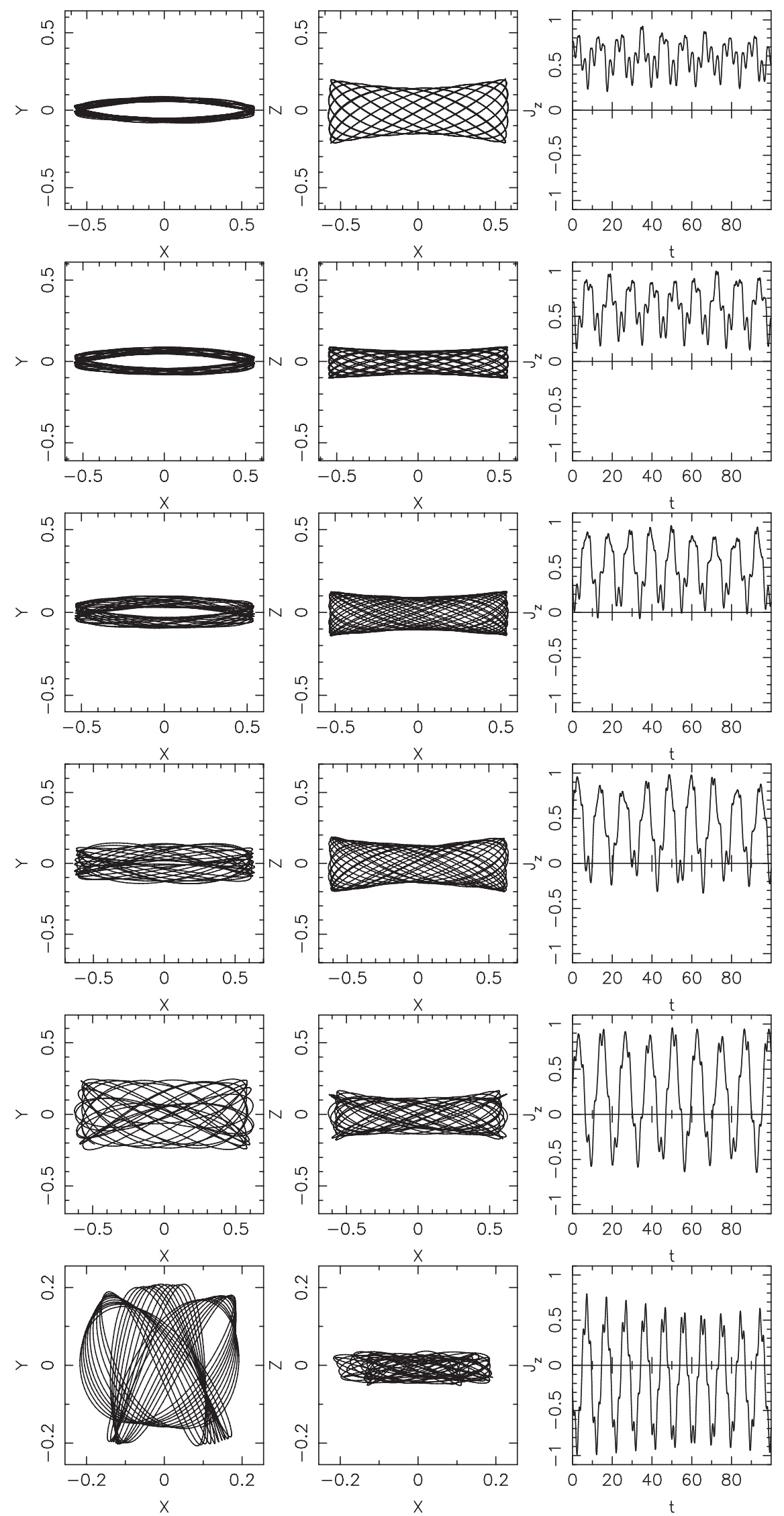

Figure 1. Left to right: $x-y$ and $x-z$ projections and normalized angular momentum $J_{z}(t)$ for six orbits from Model A that satisfy the condition $\left|\Omega_{\phi} / \Omega_{R}-1 / 2\right|<10^{-3}$ (standard definition for $\mathrm{x} 1$ orbits). The orbit in the topmost row is closest to the closed periodic parent $\mathrm{x} 1$ orbit, while orbits in successive rows get farther and farther from the parent orbit. Orbits have a narrow range of Jacobi integral $-0.89<E_{\mathrm{J}}<-0.83$. 


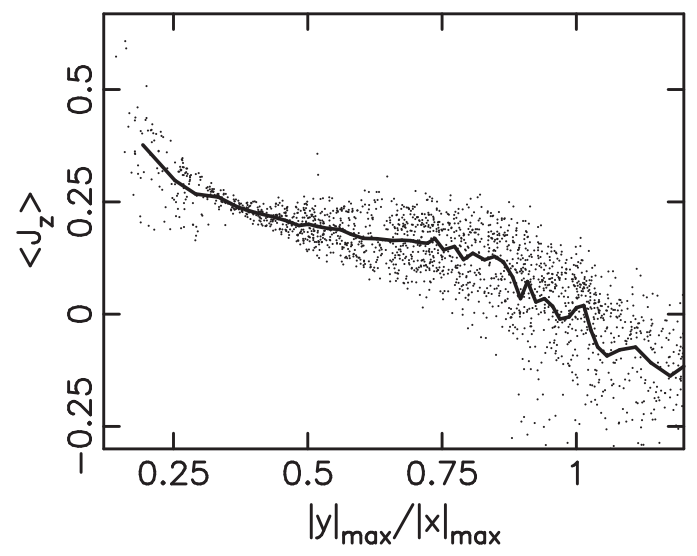

Figure 2. Each dot represents the time average of the normalized angular momentum $\left\langle J_{z}\right\rangle$ for a single orbit as a function of $|y|_{\max } /|x|_{\max }$ (see text for details). Dots are plotted for all orbits in Model A that were visually classified as $\mathrm{x} 1$ or box (but resonant 3:-2:0 boxlets were excluded). The solid line is the mean value of the distribution represented by dots.

simulations are boxes with little net rotation in the bar frame, unlike their prograde parent $\mathrm{x} 1$ orbit.

Figure 1 shows six different orbits from the pure $N$-body bar (Model A) computed in the frame of reference co-rotating with the bar. On each row we show two projections $(x-y$ and $x-z)$ and the angular momentum as a function of time $J_{z}(t)$, for a single orbit. $J_{z}(t)$ is normalized relative to its maximum absolute value over the entire orbit. The top two rows show orbits near the closed periodic $\mathrm{x} 1$ parent (it is difficult to find strictly periodic $\mathrm{x} 1$ orbits in an $N$-body simulation). For these two orbits we see that the angular momentum $J_{z}$ oscillates between two positive values throughout its orbit. The next three rows show orbits that travel increasingly farther from the parent $\mathrm{x} 1$ orbit. For these orbits $J_{z}(t)$ does not remain positive but becomes negative for those portions of the orbit when the motion is retrograde. The examples in Figure 1 demonstrate that as orbits deviate farther from the periodic $\mathrm{x} 1$ parent (i.e., as their extent in the $y$ direction increases) they spend more and more time moving retrograde. In fact, such orbits are found to be the primary building blocks of the central parts of orbitbased bar models studied by Patsis \& Katsanikas (2014b).

One way to quantify the deviation from the parent $\mathrm{x} 1$ orbit is to measure the extent of an orbit in the $y$ direction relative to its extent in the $x$ direction. We do this by computing the ratio of the maximum absolute $y$ value attained over the orbit, $|y|_{\max }$, relative to the maximum absolute $x$ value attained over the orbit, $|x|_{\max }$. Based on the visual classification of orbits, we find that classical $\mathrm{x} 1$ orbits have $|y|_{\max } /|x|_{\max }<0.35$ (they are significantly more elongated along the $x$-axis than along the $y$-axis).

Figure 2 shows $\left\langle J_{z}\right\rangle$ (the time average of the angular momentum $J_{z}$ normalized to its maximum value) as a function of $|y|_{\max } /|x|_{\max }$. Each dot represents an orbit in Model A that was classified as $\mathrm{x} 1$ or box (we exclude the resonant boxlet orbits that are discussed in the next section). The solid line is the mean value of the distribution represented by dots. Orbits with the smallest values of $|y|_{\max } /|x|_{\max }$ have the highest net angular momentum and are classical $x 1$ orbits. As orbits get thicker in $y$, this average angular momentum decreases. For reference, an orbit with constant angular momentum would have $\left\langle J_{z}\right\rangle= \pm 1$; the orbits in the top two rows of Figure 1 have $\left\langle J_{z}\right\rangle=0.6$, while the orbit in the bottom row of Figure 1 has $\left\langle J_{z}\right\rangle=-0.09$. Only a small fraction of orbits have both the high $\left\langle J_{z}\right\rangle$ and the small $|y|_{\max } /|x|_{\max }$ that are characteristic of classical $x 1$ orbits. In fact, from Figure 2 we see that most orbits have $\left\langle J_{z}\right\rangle<0.25$ and a significant fraction have negative $\left\langle J_{z}\right\rangle$, implying that they spend more time traveling retrograde than prograde (for example, the orbit in the last row of Figure 1).

We illustrate this point further by launching orbits from box orbit initial conditions in a prolate triaxial ellipsoid with the Dehnen density profile (Section 2.2), with shape parameters close to that of the $N$-body bar in Model A $(c / a=0.4, b / a=0.48, T=0.916, \gamma=0.1) . \quad$ In Figure 3 each row shows two orbits launched from the same initial conditions in a stationary Dehnen model (three left columns) and in the Dehnen model with a fast pattern speed $\left(R_{\mathrm{CR}} / r_{1 / 2}=1.1\right.$, where $r_{1 / 2}$ is the half-mass radius of the model; three right columns). For each orbit we show projections of the orbit $(x-y, x-z)$ and its angular momentum with time $\left(J_{z}(t)\right)$. The orbits in the top row were launched very close to the long $(x)$ axis of the model. On the left is a long-axis orbit (parent of the box family) with no net angular momentum $J_{z}$ or $J_{x}$. The three right-hand columns show what happens to this orbit in a rotating frame: the Coriolis force now causes the orbit to loop around the center in an anticlockwise sense, and hence $J_{z}(t)$ becomes strictly positive; i.e., in the rotating frame this orbit has become an "x1 orbit." In the second row the three left columns show a standard box orbit in a stationary potential while the three right columns show the same orbit in the rotating frame. This quasi-periodic orbit behaves in a manner identical to the "thick x1" orbits from Model A (e.g., in rows 4 and 5 of Figure 1). (The box orbits in the third and fourth rows of Figure 3 will be discussed later.)

The differences seen in the shapes and angular momentum distributions of the orbits in the stationary potential (three lefthand columns) and in the rapidly rotating potential (three righthand columns) are entirely a consequence of the pseudoforces in the rotating frame. A comparison of orbits in the first two rows of this figure with the $\mathrm{x} 1$ family orbits drawn from the $\mathrm{N}$ body bar in Figure 1 shows that both box orbits and $\mathrm{x} 1$ orbits are parented by the linear long-axis orbit. Figure 10 of SS04 gives very clear examples of quasi-periodic orbits parented by an $\mathrm{x} 1$ orbit, as well as orbits that they refer to as "fat $\mathrm{x} 1$ " (but that we call boxes). Despite their visual difference (classical $\mathrm{x} 1$ versus box-like orbits), $\mathrm{SS} 04$ show that these orbits form a continuous sequence in an SoS (e.g., upper panel of Figure 9 in SS04). We discuss this further in Section 4.2.1.

To summarize, it is well known that the $\mathrm{x} 1$ orbit in bars is the same as the parent of the box family in rotating triaxial potentials (Schwarzschild 1982; Martinet \& de Zeeuw 1988). What is less well appreciated is that in bars, as in triaxial ellipsoids, the dominant bar-supporting family is box-like with little angular momentum about the short axis, unlike their periodic $\mathrm{x} 1$ parent, which rotates prograde.

\subsubsection{Boxlets: Fish, Pretzels, and Banana Orbits}

Miralda-Escude \& Schwarzschild (1989) and Merritt \& Valluri (1999) showed that when an integrable triaxial potential is perturbed, e.g., by the introduction of a cusp or a central point mass, almost all orbits that originate from "box-like" initial conditions (i.e., launched with zero initial velocity from an equipotential surface) become either resonant or chaotic. Resonant orbits are easily identified by frequency mapping. In 

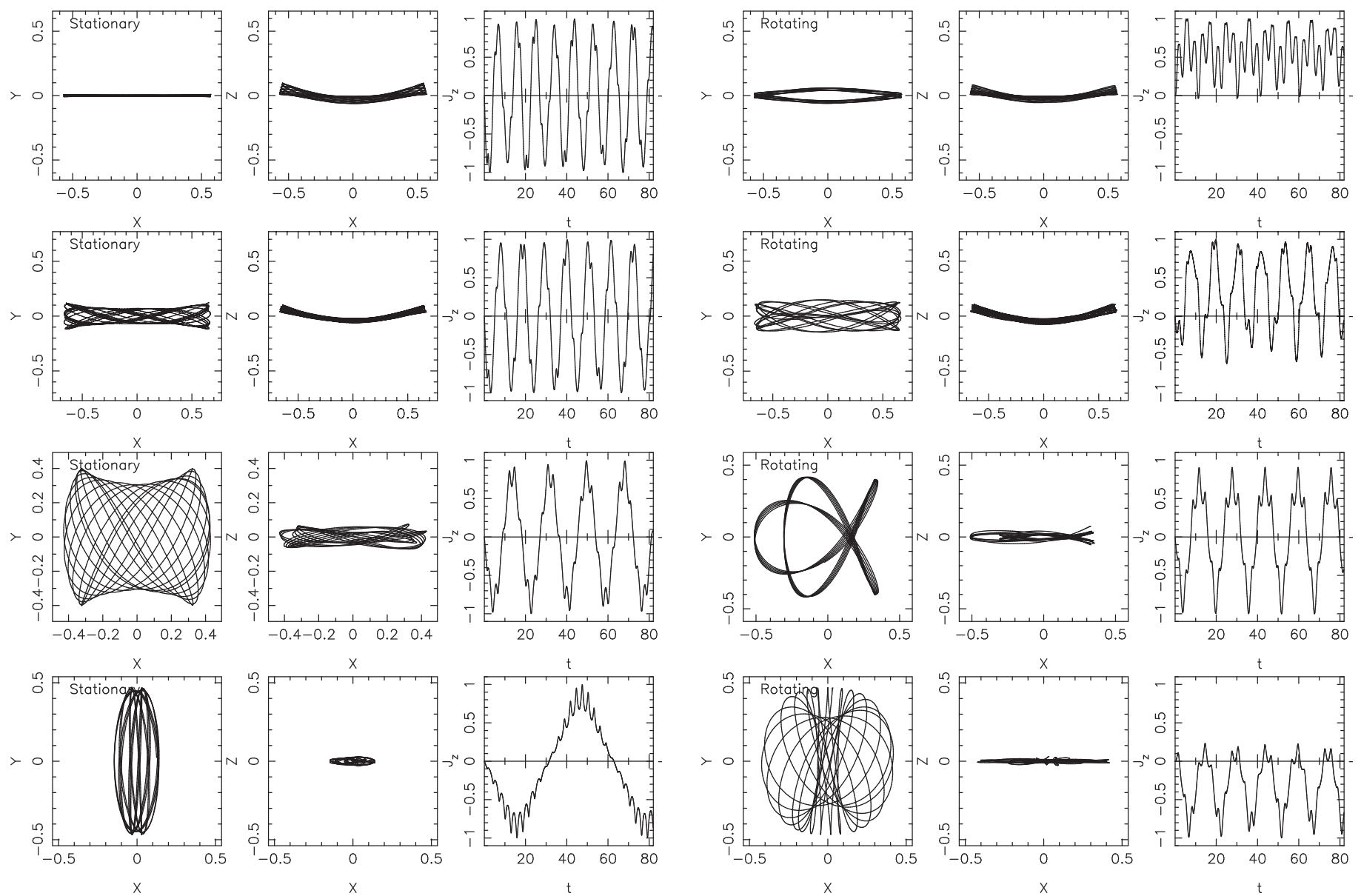

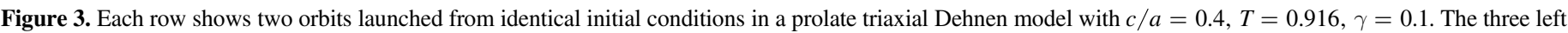

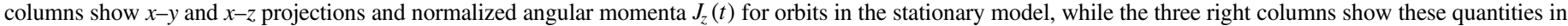

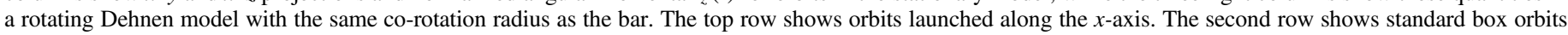

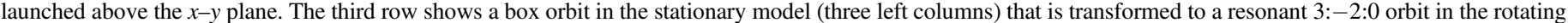
frame. The fourth row shows an orbit launched as close to the $y$-axis as the orbit in the first row is from the $x$-axis (the $y$-axis orbit itself is unstable).

stationary triaxial potentials they include the well-known 3:0: -2 "fish" resonance and the 4:-3:0 "pretzel" resonance-both of which are absent in our rapidly rotating Dehnen model and $N$-body bars. Commonly found resonant orbits in the rotating Dehnen model are shown in the third row of the three right columns of Figure $3\left(\Omega_{x}: \Omega_{y}: \Omega_{z}=3:-2: 0\right.$, fish/pretzel) and in the first and second rows of the three right columns of Figure 3 (the "banana" resonance). A few other resonances are seen in the frequency maps in Figure 12.

Our examination of orbits from the $N$-body bar showed that they can also be associated with resonances. Figure 4 shows examples of resonant orbits found in the $N$-body bar. Orbits in the first three rows are all characterized by $\Omega_{x}: \Omega_{y}: \Omega_{z}=3:-2: 0$ and look like fish/pretzels (and correspond to the same resonance as in the third row of Figure 3). The fourth row shows an orbit associated with "x1-banana" resonance $\left(\Omega_{x}: \Omega_{y}: \Omega_{z}=2:-2:-1\right){ }^{7}$ and the fifth row is an "x1-antibanana" (Miralda-Escude \& Schwarzschild 1989; Pfenniger \& Friedli 1991), which satisfies the same resonance conditions as a banana orbit, but passes through $x=0, z=0$ (the automatic classification code is unable to distinguish between banana and anti-banana, which are treated as one group). As pointed out

\footnotetext{
Note that in some papers on bars, a family of orbits that lie close to the fourth and fifth Lagrange points of the bar are also referred to as "banana" (Athanassoula 1992) but are not members of this resonant family.
}

previously by Pfenniger \& Friedli (1991), the banana and antibanana orbits are vertical bifurcations of the $\mathrm{x} 1$ family (as can be seen from their $x-y$ projections in the left-hand column). These orbits are referred to as the x $1 \mathrm{v} 1$ family by Patsis et al. (2002). Orbits like those in the second row of Figure 3 are boxy-bananas: they have a banana shape in $x-z$ associated with 2:0:-1 resonance. The third and fourth columns of Figure 4 show that like box orbits in triaxial potentials, the sign of $J_{z}$ changes at each extremum, implying that the orbits have little or no net angular momentum about the $z$-axis, despite being parented by $\mathrm{x} 1$ orbits. The frequency maps in Figure 13 show several other resonances. The presence of resonant boxlet orbits was noted by SS04 (see their Figure 13), especially at high $E_{\mathrm{J}}$ values.

Vertical bifurcations of the $\mathrm{x} 1$ orbit associated with the 2 : $-2:-1$ resonance may be responsible for the "X-shaped" structures seen in buckled edge-on bars (Patsis et al. 2002; Athanassoula 2005). Patsis \& Katsanikas (2014a, 2014b) also present a dynamical mechanism for building X-shaped peanuts with families of periodic orbits that are not bifurcations of $\mathrm{x} 1$ orbits. It was suggested by Portail et al. (2015) that a resonant family of $\Omega_{x}: \Omega_{y}: \Omega_{z}=3: 0:-5$ "brezel" orbits (bottom row of Figure 4) are the backbone of the "X-shaped" structures in their made-to-measure $N$-body bar models, but we found only 88 "brezels" (1.5\% of the bar orbits) in both Models A and B. A 

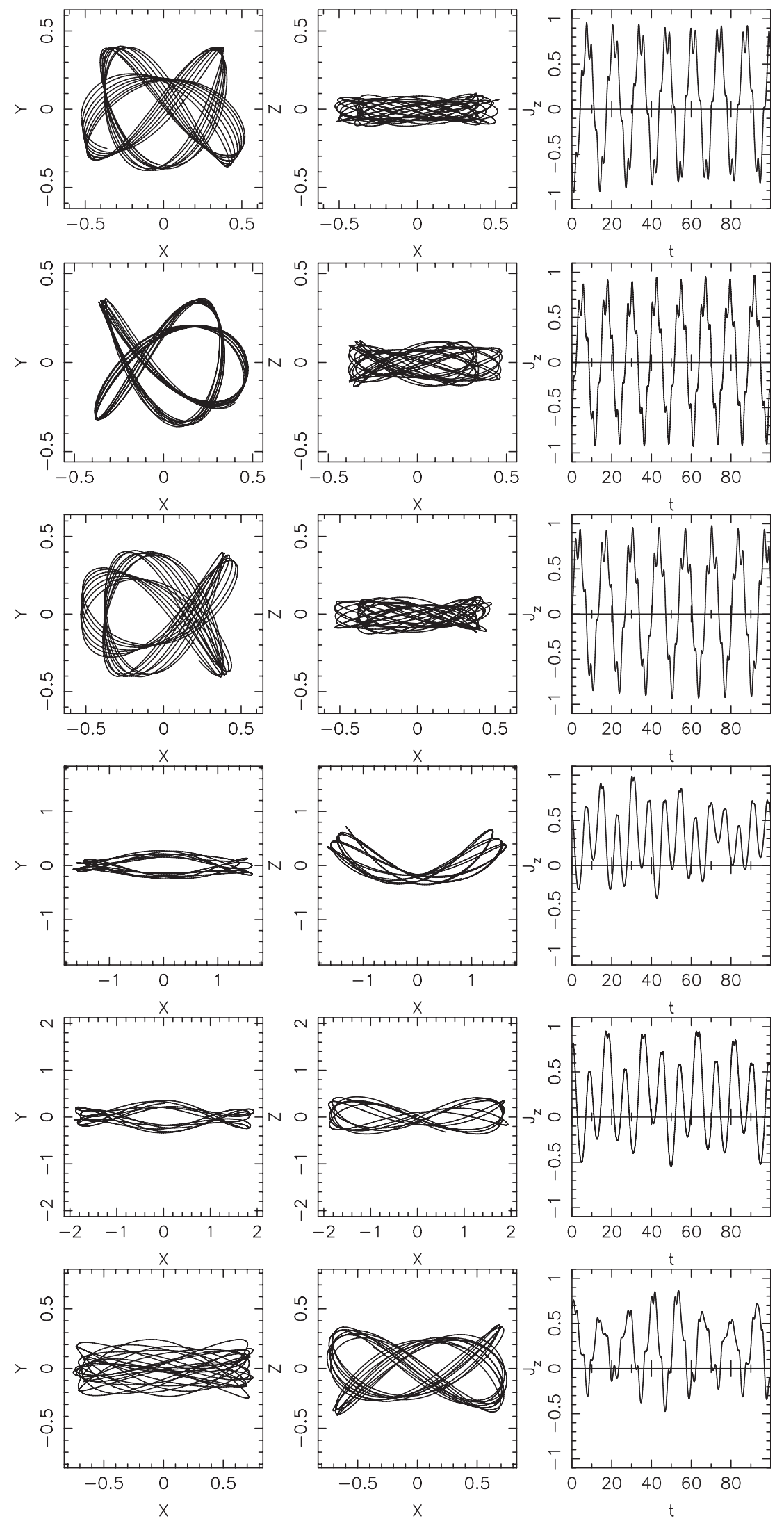

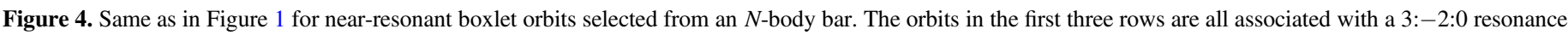

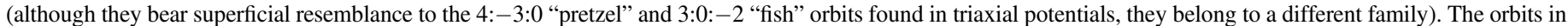

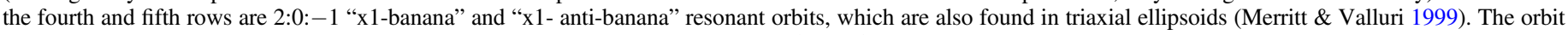
in the sixth row is classified by our automatic classifier as a resonant boxlet with $\Omega_{x}: \Omega_{y}: \Omega_{z}=3: 0:-5$. 
more detailed analysis of the orbits contributing to the X-shape will be discussed in a future work (C. Abbott et al. 2016, in preparation).

\subsection{Long-axis Tube Orbits}

De Zeeuw (1985a) showed that for integrable triaxial potentials two types of long-axis tubes exist, the inner longaxis tubes and the outer long-axis tubes. This family is "parented" by closed 1:1 periodic orbits that lie in the $y-z$ plane with angular momentum along the $x$-axis. Heisler et al. (1982) studied the stability of these periodic orbits and found that they are stable to figure rotation, but the Coriolis force tips them about the $y$-axis in a direction that depends on the sign of $J_{x}$. Two such stable periodic orbits exist rotating clockwise and anticlockwise about the $x$-axis, the orbits with positive $J_{x}$ are tipped clockwise about the $y$-axis, while orbits with negative $J_{x}$ are tipped anticlockwise about the $y$-axis. These orbits were termed "anomalous" by van Albada et al. (1982) since they both acquire retrograde motion about the $z$-axis in the rotating frame as a result of being tipped. Since the long-axis tube family is "parented" by anomalous orbits, they too are expected to be stable and "tipped" about the $y$-axis in a rotating frame, and may acquire some retrograde angular momentum about the $z$-axis.

Figure 5 shows examples of inner long-axis tubes (top two rows) and outer long-axis tubes (third and fourth rows) selected from bar Model A. The second column ( $y-z$ projection) shows that these orbits circulate about the long $(x)$ axis. The rightmost column shows the angular momentum $J_{x}$ about the $x$-axis, which, as expected, is strictly positive or strictly negative. An inspection of the first column shows that the orbits are tipped about the $y$-axis exactly as predicted by Heisler et al. (1982), further supporting our claim that bars and rotating triaxial ellipsoids have fundamentally similar orbital building blocks. As far as we are aware, previous studies of self-consistent orbits in three-dimensional $N$-body bars (Voglis et al. 2007) have not found long-axis tubes, suggesting that their existence could be sensitive to bar shape and the details of its formation mechanism.

\subsection{Short-axis Tubes, $x 2$ and $x 4$ Orbits}

Short-axis $(z)$ tubes constitute an important family in oblatetriaxial ellipsoids but are somewhat less important in more prolate systems. Short-axis tubes are parented by closed periodic $1: 1$ orbits that lie in the $x-y$ plane and circulate about the $z$-axis. These orbits are known to be stable to figure rotation (de Zeeuw \& Merritt 1983). However, Deibel et al. (2011) found that as the pattern speed of a triaxial figure was increased, prograde short-axis tubes were replaced by retrograde ones. This was also found by Martinet \& de Zeeuw (1988) for loop orbits in the $x-y$ plane of a rotating triaxial ellipsoid and occurs because tube/loop orbits launched prograde "fall behind" the figure, becoming retrograde as the pattern speed increases.

Periodic $\mathrm{x} 2$ and $\mathrm{x} 4$ orbits (like the classical $\mathrm{x} 1$ orbit) satisfy the condition $\Omega_{R}: \Omega_{\phi}=2: 1$. In the characteristic diagrams (e.g., Sellwood \& Wilkinson 1993) the prograde $x 2$ family is found at low $E_{\mathrm{J}}$ and is elongated along the $y$-axis of the bar, while the $\mathrm{x} 4$ family is retrograde and is found over a wide entire range of $E_{\mathrm{J}}$ values. Both the $\mathrm{x} 2$ and $\mathrm{x} 4$ orbits are elongated along the $y$ axis at small $E_{\mathrm{J}}$. The $\mathrm{x} 4$ family is elongated along the $y$-axis at small $E_{\mathrm{J}}$ but becomes rounder at large $E_{\mathrm{J}}$. Since $\mathrm{x} 2$ and $\mathrm{x} 4$ orbits are planar periodic orbits, they are not expected to be found in significant numbers in an $\mathrm{N}$-body model, but they do parent families of prograde and retrograde short-axis tubes, which can be easily identified if they exist. Henceforth, we refer to all nonplanar orbits with a fixed sign of angular momentum about the short axis as " $z$-tubes."

The visual classification of 20,000 orbits in Model A and Model B identified $\sim 1.5 \%$ of bar orbits in Model $\mathrm{A}$ as retrograde $z$-tubes, but none of the orbits in this model were found to be prograde $z$-tubes. The top two rows of Figure 6 show examples of retrograde $z$-tubes from Model A. The fact that we were unable to find a single example of a prograde $z$ tube orbit is consistent with the findings of Sparke \& Sellwood (1987) and Voglis et al. (2007), who only found retrograde x4 orbits in their $N$-body bar models. x 2 orbits (and orbits parented by them) are expected from the locations of the inner Lindblad resonance and the co-rotation resonance in Model A; however, this family appears to be severely underpopulated. A significant decline in prograde $z$-tubes at high pattern speeds is, however, predicted by Martinet \& de Zeeuw (1988) and Deibel et al. (2011).

We examined whether it was possible to generate $\mathrm{x} 2$ and $\mathrm{x} 4$ orbits from perturbations to a linear $y$-axis orbit in the same way as it was possible to generate the $\mathrm{x} 1$ orbit from the linear $x$ axis orbit. The $y$-axis linear orbit is known to be unstable (Adams et al. 2007); hence, launching orbits from the $y$-axis of the Dehnen model yielded unstable chaotic orbits. An example of an orbit launched from the "stationary start space" (i.e., with no initial velocity) near (but not on) the $y$-axis is shown in the fourth row of Figure 3. In the stationary frame (three left columns) the orbit is a stable box elongated along the $y$-axis. In the rotating frame (three right columns) this orbit becomes an even thicker box but now with $\left\langle J_{z}\right\rangle<0$, similar to the orbit in the last row of Figure 1. If $\left|J_{z}\right|$ was smaller than a minimum value, box-like orbits elongated along the $y$-axis always resulted. It was only possible to generate retrograde $\mathrm{x} 4$ orbits by launching an orbit from near the $y$-axis with a substantial $\left|J_{z}\right|$. All the retrograde $z$-tubes in Model A are found at small values of $E_{\mathrm{J}}$ and are more elongated along the $y$-axis than along the $x$-axis.

In Model B, however, we do find prograde and retrograde $z$ tube orbits. Some are more elongated along the $y$-axis, while others are more elongated along the $x$-axis than along the $y$ axis. We defer a discussion of this to Section 4.1, where we argue, based on the work of Brown et al. (2013), that this new population of prograde $z$-tubes is a result of the growth of the $\mathrm{SMBH}$ in the bar, which induces angular momentum redistribution.

Figure 6 shows three prograde $z$-tubes from Model B. In the third row the orbit is elongated along the $y$-axis (i.e., it is parented by the periodic $\mathrm{x} 2$ orbit); in the fifth row it is elongated along the $x$-axis, and the fourth row shows a transitional orbit. We assert that all these orbits belong to the same family since they must have significant initial net angular momentum to avoid becoming boxes, in contrast with $\mathrm{x} 1$ orbits, which are related to boxes and derive their prograde motion from the Coriolis forces.

\subsection{Precessing Keplerian Orbits (PKOs) around an SMBH}

Previous studies of orbits in triaxial and axisymmetric potentials have shown that as one approaches the massive 

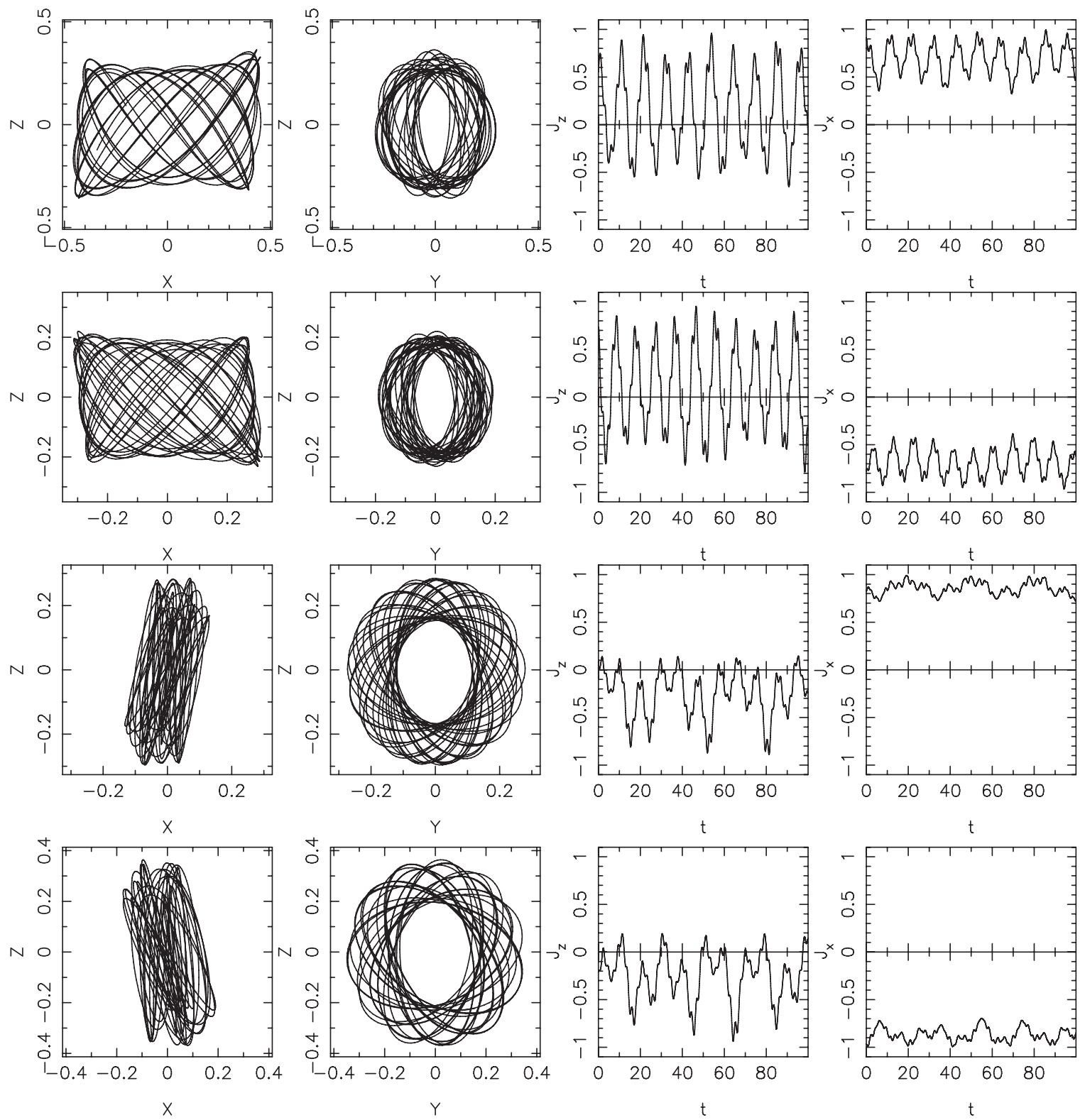

Figure 5. Left to right: $x-z, y-z$ projections and angular momenta as a function of time $\left(J_{z}(t), J_{x}(t)\right)$ for long-axis tube orbits selected from Model A. Orbits in the top two rows are inner long-axis tubes, while the orbits in the lower two rows are outer long-axis tubes. Owing to the Coriolis forces, the orbit is tipped clockwise (anticlockwise) about the $y$-axis when $J_{x}(t)$ is positive (negative). Notice that the two outer long-axis tubes are tipped so far about the $y$-axis that they acquire significant net negative $J_{z}$, as predicted by Heisler et al. (1982).

central point mass in the region of the potential where the mass of stars is comparable to the mass of the SMBH, orbits begin to resemble Keplerian ellipses, which are perturbed by the largescale triaxial or axisymmetric stellar potential and therefore precess slowly (Sridhar \& Touma 1999; Poon \& Merritt 2001; Merritt \& Vasiliev 2011; Li et al. 2015). Orbits associated with black holes include "saucers," "pyramids," and a variety of resonant families (Merritt \& Valluri 1999; Merritt \& Vasiliev 2011). In this work we will refer to these collectively as PKOs.

The spatial resolution of our simulations was high enough $^{8}$ that random sampling of the distribution function of Model B yielded a small number of PKOs in the vicinity of

\footnotetext{
8 Black hole softening length $\epsilon=0.001$ units ( $1 \mathrm{pc}$ in physical units); see Brown et al. (2013) and SS04 for more details.
}

the black hole. Figure 7 shows examples of PKOs that were found in Model B. Each row shows a single orbit: the three panels on the left show the orbit integrated for about 20 orbital periods, ${ }^{9}$ while the three panels on the right show the projected density distribution of the orbit integrated over hundreds of orbital periods (for $t=1000$ units). Notice that in the short integrations (left three columns) the orbits in the top three rows look very similar to each other: they are all slowly precessing ellipses. However, the projected density distributions of the same orbits show that they are morphologically quite different from each other. The orbit in the top row is a box (although $J_{z}(t)$ and $J_{x}(t)$ are not shown, it also

\footnotetext{
9 Orbits were integrated with NAG Mathematical Libraries subroutine D02CJF, a variable-order, variable-step implementation of Adams's method, which is very accurate.
} 

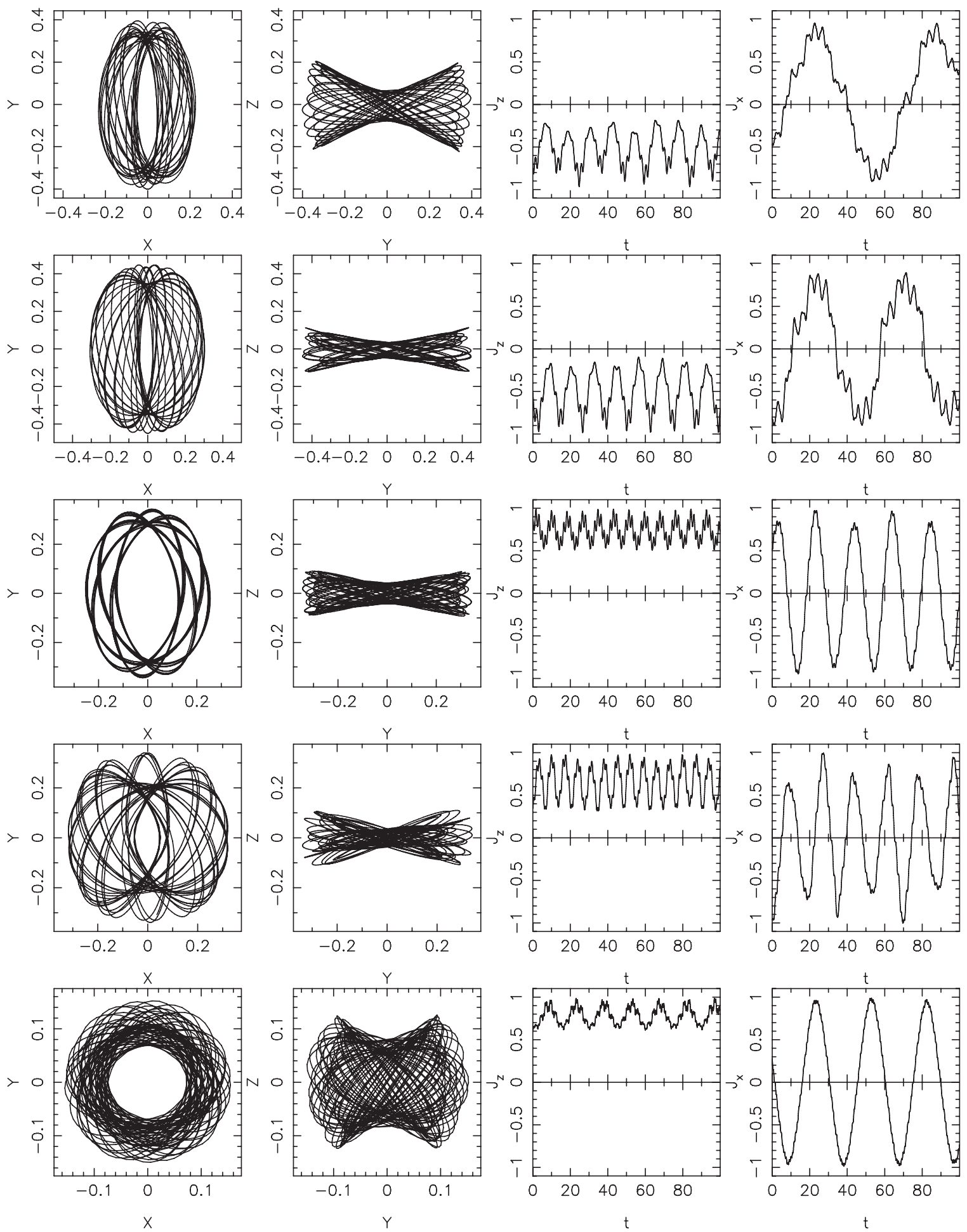

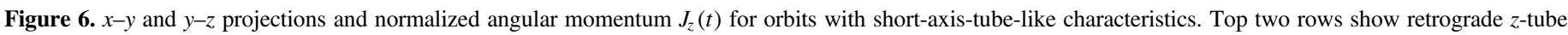
orbits selected from Model A (this model has no prograde $z$-tubes). The last three rows show prograde $z$-tube orbits all from Model B.

has no net angular momentum about either axis), the second row shows a short-axis $(z)$ tube for which $J_{z}(t)>0$, and the third row shows a resonant short-axis tube. The bottom row shows a long-axis tube $\left(J_{x}(t)<0\right)$ that is tipped anticlockwise about the $y$-axis. (The orbits in the last two rows appear to pass through the origin, but zoomed-in plots show that they do not.) In total, 113 orbits (1.8\% of bar orbits) in Model B were found to be PKOs. At the very small radii at which these orbits are found, the effects of the centrifugal forces from the rotating pattern should be quite small, but the Coriolis forces are large enough (because the velocities are high) to produce effects similar to those seen on orbits at larger radii. A detailed study of the effects of figure rotation on these nearly Keplerian orbits is beyond the scope of this paper and will be investigated in a future paper. 

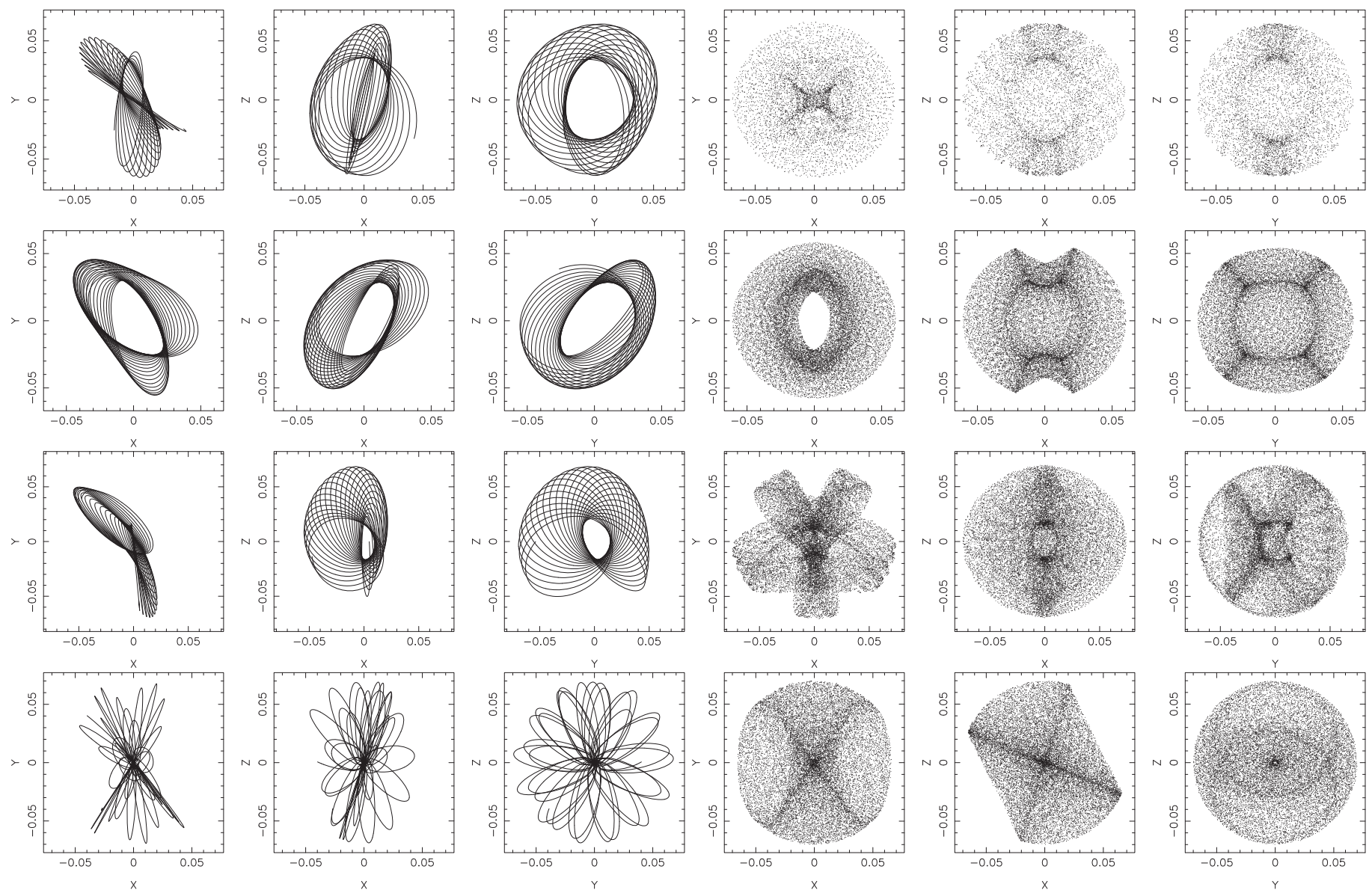

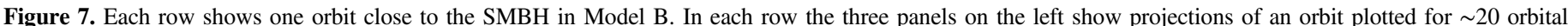

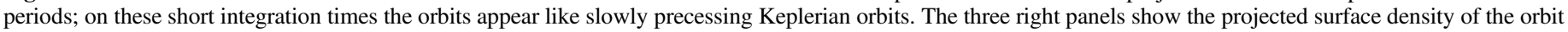

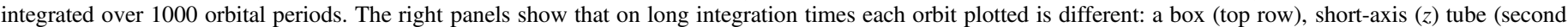
row), resonant $z$-tube (third row), and $x$-tube (fourth row).

Table 1

Visual Classification vs. Automatic Classification ${ }^{\mathrm{a}}$

\begin{tabular}{|c|c|c|c|c|c|c|c|c|}
\hline Model & Classifier & $x$-tubes & $\mathrm{x} 4+\mathrm{x} 2+z$-tubes & $\mathrm{x} 1+$ bananas & Boxes & $3:-2: 0$ & Chaotic & Disk \\
\hline \multirow{3}{*}{ A } & Visual (C.A.) & $369(7.0 \%)$ & $90(1.6 \%)$ & $407(7.2 \%)$ & $3831(67.5 \%)$ & $212(3.7 \%)$ & $740(13.0 \%)$ & $4324(\mathrm{~N} / \mathrm{A})$ \\
\hline & Auto & $494(8.5 \%)$ & $31(0.5 \%)$ & $171(3.0 \%)$ & $3707(65.3 \%)$ & $343(5.9 \%)$ & $1049(18.5 \%)$ & $4205(\mathrm{~N} / \mathrm{A})$ \\
\hline & Visual (C.A.) & $305(5.0 \%)$ & $81(1.3 \%)$ & $360(5.9 \%)$ & $3874(64.0 \%)$ & $45(0.7 \%)$ & $1392(23.0 \%)$ & $3706(\mathrm{~N} / \mathrm{A})$ \\
\hline B & Auto & $250(4.0 \%)$ & $147(2.3 \%)$ & $196(3.1 \%)$ & $4197(66.7 \%)$ & $119(1.9 \%$ & $1395(22.2 \%)$ & $3696(\mathrm{~N} / \mathrm{A})$ \\
\hline
\end{tabular}

Note.

${ }^{a}$ In each column the integer indicates the number of orbits, while the quantity in parentheses is the percentage of that type in the bar only.

\section{ORBIT POPULATION STATISTICS AND PHASE- SPACE DISTRIBUTION}

\subsection{Comparison of Automatic and Visual Classification}

Table 1 compares the overall orbit fractions obtained by both classification methods (visual and automatic) for both models. The integer in each column represents the total number of orbits in that family (from a total of 10,000 orbits in each model), while the percentage of bar orbits (i.e., excluding disk orbits) contributed by this family is given in parentheses. Note that for Model B we exclude the 113 visually classified "PKO" orbits since the automated method is unable to distinguish these from boxes, long-axis tubes, and short-axis tubes at large radii.
In Figures 8, 10, and 11, and Table 1 we present $\mathrm{x} 1$ orbits, 3: $-2: 0$ orbits, and boxes separately but remind readers that they are all members of the box orbit family.

The biggest difference between the automatic and visual classification is the fraction of orbits that are classified as "chaotic" or "x $1 / \mathrm{x} 1+$ banana." Many of the orbits that are visually classified as " $\mathrm{x} 1 / \mathrm{x} 1+$ banana" are automatically classified as chaotic (and, as we see in Figure 8, tend to lie toward the outer part of the bar). In addition, while 90 orbits were visually classified as retrograde $z$-tube orbits, only 31 were automatically classified as such. Examination of the remaining 60 showed that they were also identified as chaotic by the automatic classifier. 

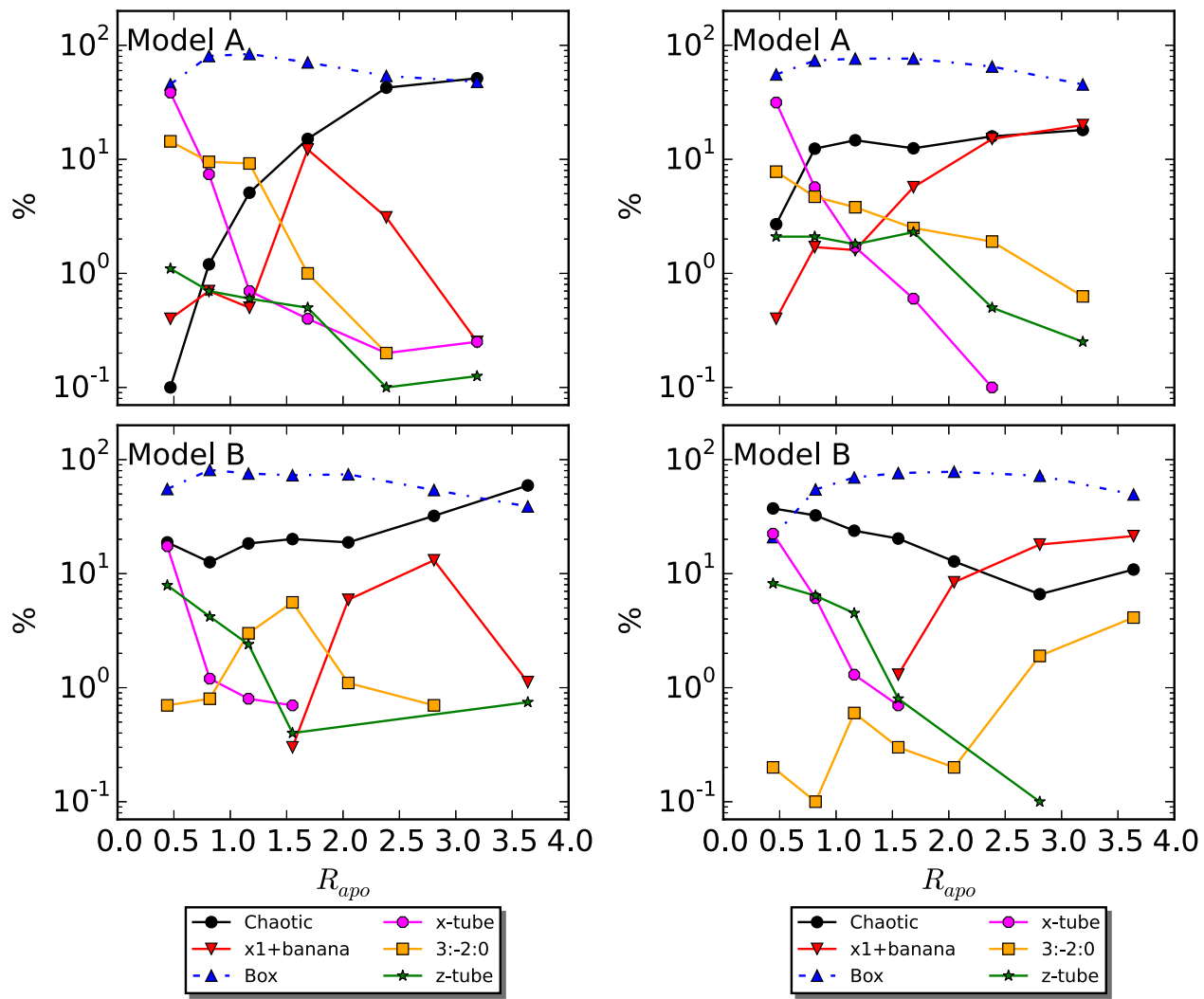

Figure 8. Percentage of orbits in various orbit families as a function of $R_{\text {apo }}$ as determined via automated orbit classification (left) and visual classification (right), in Model A (top) and Model B (bottom). Radial bins have equal number of orbits per bin and only particles with $R_{\text {apo }}<4 \mathrm{kpc}$ are plotted. The percentages within a bin sum to $100 \%$ (note that the ordinate is logarithmic). If the percentage of orbits in a given family falls to zero, no symbol is plotted.

In Figure 8 we compare how the orbit populations in Models A and B change with $R_{\text {apo }}$ (the apocenter radius of orbits in the $x-y$ plane) and how the orbit population at each radius depends on the classification scheme.

We distribute bar orbits into 6 bins in $R_{\text {apo }}<4 \mathrm{kpc}$. Figure 8 shows the percentage of orbits (on a logarithmic scale) in each bin as a function of $R_{\text {apo }}$ in each of the main families (as indicated by the legends). The left-hand column shows percentage of orbits in each radial bin as determined by the automated classification scheme, while the right-hand column shows the same plot using visual classification. At all radii the orbits classified as boxes (blue triangles, connected by blue dotdashed lines) dominate the population in both models. The fractions of boxes obtained by both methods are also nearly identical. The $x$-tube orbits (pink circles connected by pink lines) decline with radius in both models, and there are slightly more of them in Model A than in Model B (both classification methods yield similar fractions of these orbits). The short-axis tube family shown by green stars connected by solid lines is slightly more important in Model B than in Model A. The trend of the short-axis tube population with radius is similar in both classification schemes. There are somewhat more chaotic orbits (black circles connected by black lines) in the inner regions of Model B than in Model A (with both methods). Visual classification yields fewer chaotic orbits in the outer regions of both models than does the automatic classification. The fractions of $\mathrm{x} 1+$ banana orbits (red triangles connected with solid red lines) and 3:-2:0 resonant orbits (yellow squares connected with solid yellow lines) differ between automatic (left) and visual classification methods (right), but both populations are small (less than 10\%) at most radii. The biggest differences are in the outer part of the bar, where the visual classification identifies orbits as $\mathrm{x} 1$ or $3:-2: 0$, while the automated classification classifies these orbits as chaotic.

Overall Figure 8 shows that the populations in Models A and $\mathrm{B}$ resemble each other and that by both methods of classification box orbits and chaotic orbits make the most significant contribution to the overall population within the bar in both models. All other families ( $x$-tubes, $z$-tubes, $\mathrm{x} 1$ +banana, and resonant 3:-2:0) each contribute less than $10 \%$ at any radius. The overall trends with radius are similar, but there are differences of a few $(\sim 2 \%-3 \%)$ between the two models and between the numbers obtained by visual and automated classification schemes. These differences are comparable to the differences in classification obtained by two different visual classifiers. Since these differences are small and unavoidable, in the rest of this paper we use automated classification as the basis for our analysis.

\subsection{Phase-space Structure of Bars}

\subsubsection{Poincáre SoSs}

The distribution of $E_{\mathrm{J}}$ values for all the orbits in each of the two models is shown in Figure 9. Since the particles were selected at random from the simulations, each distribution is representative of the distributions of $E_{\mathrm{J}}$ values for the entire model. Five bins containing 100 orbits each were defined to lie roughly at equal intervals in $E_{\mathrm{J}}$ between the minimum value of $E_{\mathrm{J}}$ and the value at the co-rotation radius of the bar. The bin 

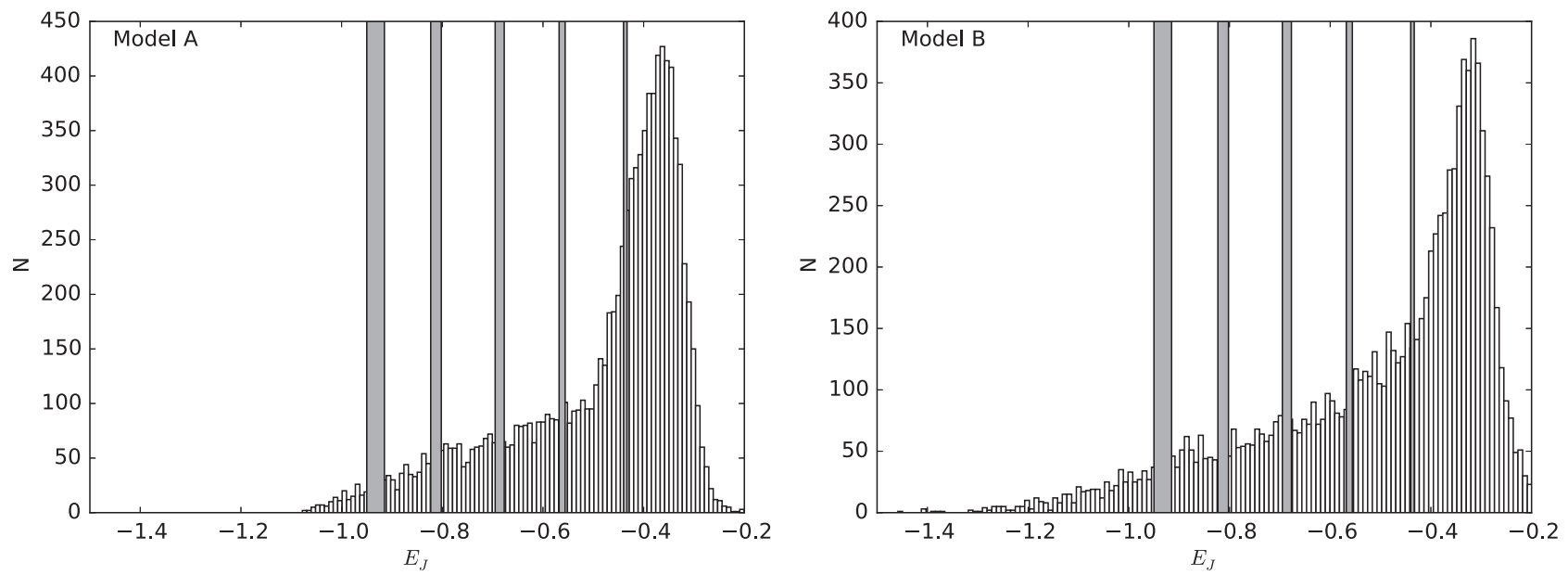

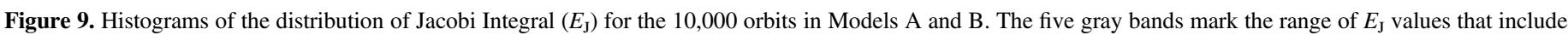
the 100 orbits plotted in the SoSs in Figure 10 and Figure 11.

limits are shown as gray bands overlying the histograms. These bins were used to select orbits to plot the SoSs that follow.

A standard way to represent the phase-space distribution of orbits in a bar is to plot a Poincáre SoS for a number of orbits at a single value of the Jacobi Integral $E_{\mathrm{J}}$. For two-dimensional bars it is customary to construct an SoS by plotting the velocity component $V_{y}$ as a function of $y$ every time an orbit intersects the $x$-axis with a negative value of $V_{x}$ (e.g., Sellwood \& Wilkinson 1993). In three-dimensional bars one might choose to restrict orbits plotted on an SoS to those that are confined to the equatorial (i.e., $x-y$ ) plane of the model (e.g., Shen \& Sellwood 2004). When orbits of a single value of $E_{\mathrm{J}}$ are plotted on an SoS, regular orbits follow thin closed or broken curves, resonant orbits form groups of islands, while chaotic orbits tend to fill larger areas.

Two problems arise in plotting an SoS for orbits from an $\mathrm{N}$ body simulation: first, all orbits occupy three spatial dimensions and are not well represented on a two-dimensional SoS; second, since orbits have a range of $E_{\mathrm{J}}$ values (see Figure 9), curves in the SoS appear fuzzy. Figure 10 show SoSs centered on five different $E_{\mathrm{J}}$ values in Model $\mathrm{A}$. The left-hand column shows $y$ versus $V_{y}$ when orbits cross the $y-z$ plane with positive $V_{x}$; the $\operatorname{SoS}$ is appropriate for $\mathrm{x} 1$ orbits (red dots), box orbits (blue dots), 3:-2:0 orbits (orange dots), and $\mathrm{x} 4$ orbits (green dots). The SoSs in the right-hand column show $V_{z}$ versus $z$ when orbits cross the $x-z$ plane with negative $V_{y}$; these coordinates are more meaningful for $x$-axis tubes (pink dots). Chaotic orbits (black dots) were plotted in the right-hand plots simply to avoid crowding.

In Figure 10 the spread in $E_{\mathrm{J}}$ (as indicated in the legends) results in few clear curves in the SoSs. Nonetheless, one can see that each orbit family occupies a slightly different region of the SoS. $x 1$ orbits (red dots) that are prograde about the $z$-axis cluster around $V_{y}=0$ at positive $y$ values (see dense red core at the bull's-eye of the SoS in Figure 10). Box orbits (blue regions) lie on oval curves surrounding the $\mathrm{x} 1$ orbits, indicating that they belong to the same sequence. (This is much more clearly seen in Figure 9 of SS04, which shows that the $\mathrm{x} 1$ orbits form the smallest ovals forming a "bull's-eye," and this sequence of ovals extends to larger radii.) Since the bigger circles (and the blue regions in our plots) can have negative $y$ values, it implies that their $J_{z}$ becomes negative as they acquire a box-like appearance. In an SoS resonant orbits appear as multiple islands. Seen from the point of view of dynamical tools like the SoS, box orbits and $\mathrm{x} 1$ orbits do belong to the same family, although they can have slightly different appearances. SS04 show that a large number of resonances of various orders can (in principle) exist in an $N$-body bar potential. However, our SoSs (and frequency maps in the next section) show that only one prominent resonant family is populated in the distribution function. The 3:-2:0 family appears in the SoS in Figure 10 as orange points that appear in multiple discontinuous islands in the region occupied by box orbits. In Figure 10 retrograde $z$-tubes (green dots) lie to the left of $y=0$, and there are no prograde (x2) orbits in this model.

Figure 11 shows SoSs after the growth of the SMBH (Model B). The most striking difference between Model B (after SMBH growth) and Model A is the appearance of prograde $z$ tube orbits (parented by the $\mathrm{x} 2$ orbit). It is worthwhile reviewing briefly the results of Brown et al. (2013), which will shed light on the appearance of these orbits. Brown et al. (2013) carried out a detailed analysis of the intrinsic velocity distributions (velocity dispersion profiles and velocity anisotropy) in the models analyzed here, as well as an axisymmetric disk in which an SMBH was grown in an identical manner. In both a barred and axisymmetric galaxy, the growth of the $\mathrm{SMBH}$ increases the depth of the central potential ("adiabatic contraction"), but the density increase was significantly higher in the barred galaxy than in the axisymmetric one. Furthermore, as matter is dragged inward in the axisymmetric galaxy, the distribution function becomes tangentially biased and inflow is limited by angular momentum conservation. However, in a time-dependent barred galaxy the inward flow of mass is enhanced by outward transport of angular momentum. This results in a higher central stellar density in the bar than in the axisymmetric model. The differences between Model A and Model B arise as a result of two effects: (a) elongated orbits, e.g., $\mathrm{x} 1$ orbits and $x$-tubes are unable to survive the growth of the central point mass, which makes the potential more axisymmetric (Deibel et al. 2011); and (b) inflow of matter from large radii with positive angular momentum results in the formation of prograde $z$-tube orbits seen in the SoS, which did not exist in the original bar. 

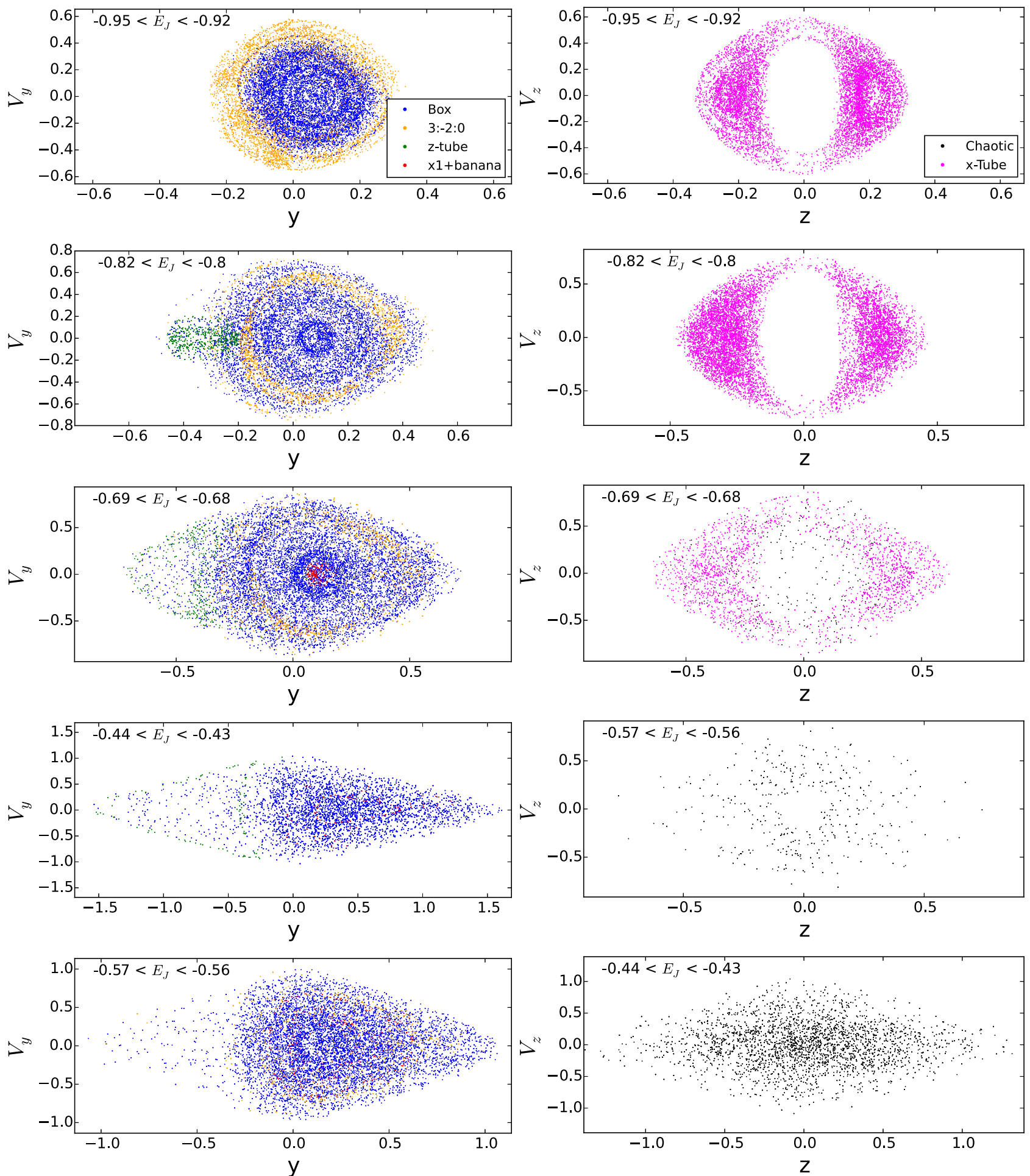

Figure 10. SoSs for orbits in Model A: left column shows $y$ vs. $V_{y}$, while the right column shows $z$ vs. $V_{z}$. Each row shows SoSs for 100 orbits in one of the five ranges in $E_{\mathrm{J}}$ (shown as gray vertical bars in Figure 9). The SoSs in the left-hand column plot x1 orbits (red points), box orbits (blue points), 3:-2:0 orbits (orange points), and $\mathrm{x} 4 / \mathrm{x} 2 / z$-tube orbits (green points), while the SoSs in the right-hand column plot only $x$-axis tubes (pink points) and chaotic orbits (black points).

\subsubsection{Frequency Maps}

An alternative way to represent the phase-space distribution function is via a "frequency map." Frequency mapping exploits the fact that most orbits in galaxies are approximately quasiperiodic (BT08); hence, a Fourier transform of their space and/ or velocity coordinates can be used to obtain the fundamental orbital frequencies that characterize each regular orbit (for a detailed description of the orbital spectral analysis method see Appendix A). A frequency map is useful for representing the phase-space structure of a large sample of orbits even if they have a wide range of energies (or $E_{\mathrm{J}}$ ). On such a map one can plot a large number of orbits that are representative of an entire distribution function (instead of just a subset at discrete values of $E_{\mathrm{J}}$ as in the case of SoSs). In an appropriate coordinate 

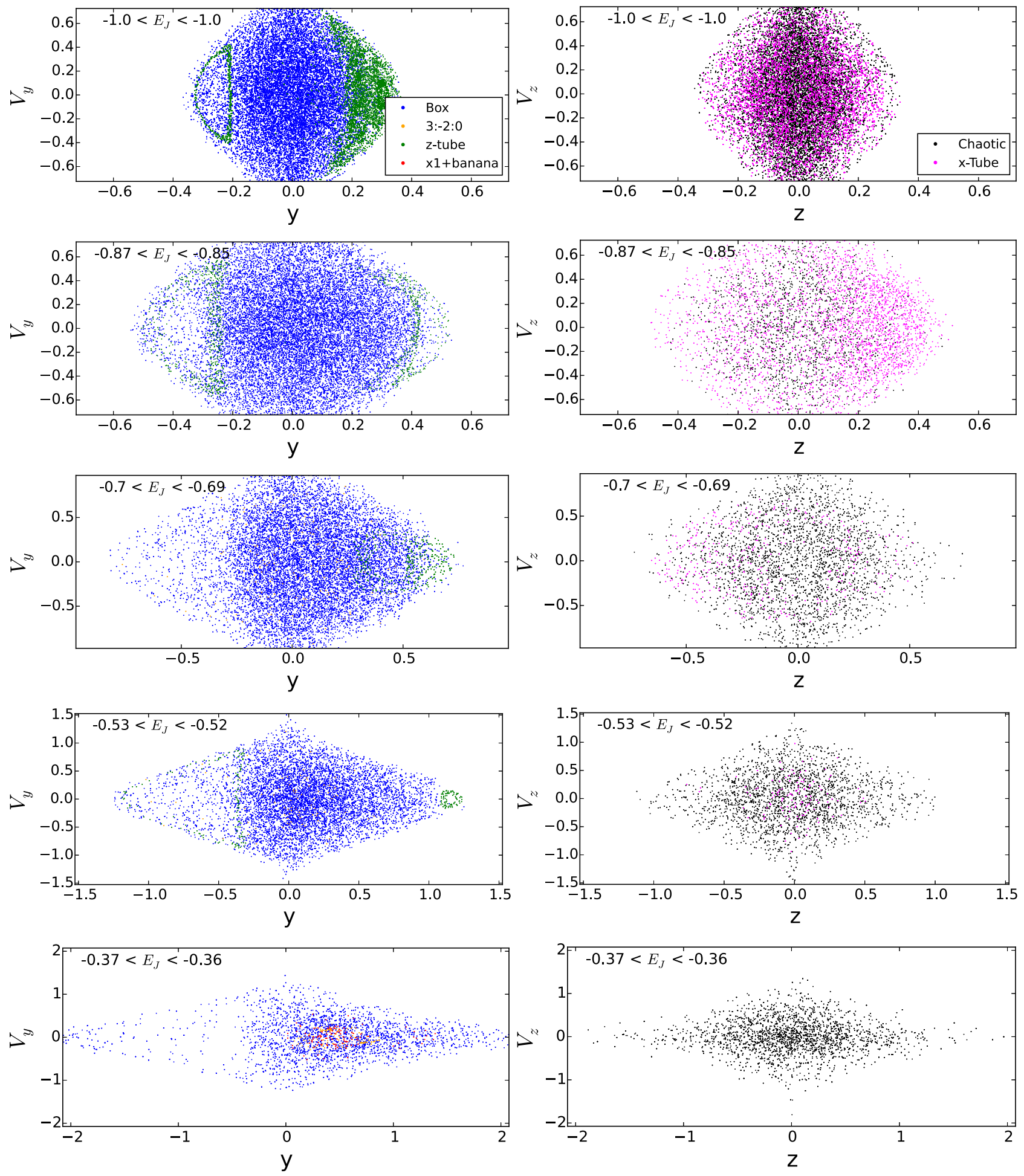

Figure 11. Same as Figure 10 but for orbits in Model B.

system, orbits belonging to different families will appear in different regions of the map, providing an easy way to visually assess the importance of various orbit families to the distribution function and the range of $E_{\mathrm{J}}$ values for which each family is important. Frequency maps are also useful for identifying different resonances and their importance to the distribution function (Valluri et al. 2010, 2012).

A frequency map is obtained by plotting pairs of fundamental orbital frequencies or ratios of such frequencies against each other. We integrated a large number of orbits in the Dehnen model at a single energy level launched from "stationary start space" initial conditions (i.e., zero initial velocity) to generate box orbits. The same initial conditions were integrated with figure rotation. Figure 12 (top) shows the three-dimensional distribution of these 3888 initial conditions, with each point given a unique color determined by its coordinates $(x, y, z)$. The RGB color indices are determined such that points that lie on the $x, y, z$ axes are red, green, and 

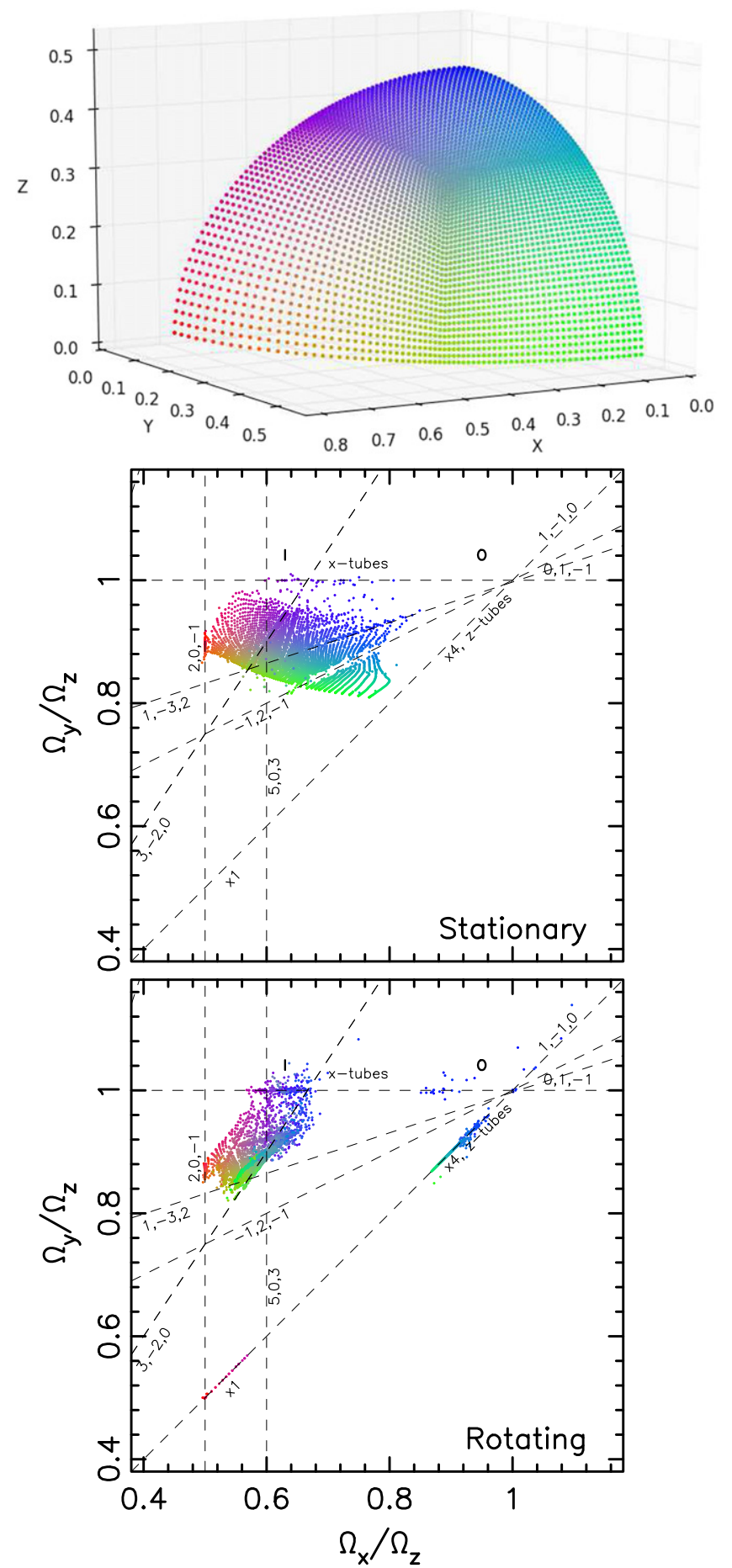

Figure 12. Top: 3888 initial positions from which the box orbits are launched with zero initial velocity (i.e., box orbit initial conditions). Each point is given a unique color (determined by its coordinate) to enable readers to map the start space to the frequency maps below. Middle: frequency map of the orbits launched from the initial conditions in the top panel in a stationary Dehnen model. Bottom: frequency map of the same initial conditions in the rapidly rotating Dehnen model. Several resonance lines are marked with dashed lines and resonance integers; others are clearly visible in the clustering of points but are not marked to avoid crowding.

blue, respectively, and the colors of other points reflect their

\footnotetext{
10 The RGB color index of a point with coordinate $x_{i}, y_{i}, z_{i}$ is given by $x_{i} / x_{\max }, y_{I} / y_{\max }, z_{i} / z_{\max }$, where $x_{\max }, y_{\max }, z_{\max }$ mark intersections of the equipotential surface with the principle axes.
}

distances from the principal axes. ${ }^{10}$ The middle panel shows the frequency map in the stationary Dehnen model, while the bottom panel shows the frequency map of the same orbits subjected to figure rotation. The colors of points in the frequency maps enable the reader to visually map points on the frequency maps to their initial launch positions in the top panel.

In the stationary Dehnen model (middle panel) most of the points lie in a fairly regular grid above the diagonal $(1,-1,0)$ resonance line and below the horizontal $(0,1,-1)$ resonance line. In the rotating Dehnen model (bottom panel) most of the points move to the left, but a few points appear along the (1, $-1,0)$ diagonal near the label "x1." Their red color indicates that they were launched from near the $x$-axis, and as expected they are converted to " $\mathrm{x} 1$ " orbits in the rotating frame. There are box-like red points associated with the $(2,0,-1)$ "banana resonance," which is bifurcation of the $\mathrm{x} 1$ family (these are the box-like orbits that also have the banana shape in $x-z$ projection, e.g., second row of Figure 3, but are launched from some height above the $x-y$ plane).

As predicted by Martinet \& de Zeeuw (1988), $z$-axis orbits (and orbits launched close to the $z$-axis, shown in blue) with adequate angular momentum generate both retrograde $z$-tubes (parented by the $\mathrm{x} 4$ family) and the long-axis tubes (parented by the stable anomalous orbits). These orbits appear as blue dots along the horizontal $(0,1,-1)$ resonance and at the top of the diagonal $(1,-1,0)$ resonance. The label "I" shows the location of the inner long-axis tubes, and the label " $O$ " shows the location of the outer long-axis tubes. Finally, orbits launched close to the intermediate $y$-axis in the rotating Dehnen model with sufficient angular momentum generate retrograde $z$-tube orbits (which appear as green dots along the $(1,-1,0)$ diagonal).

We now compare these frequency maps with those generated for the orbits drawn from $N$-body bar distribution functions (Figure 13) for Model A (left) and Model B (right). Accurate determination of fundamental orbital frequencies requires that orbits are integrated for at least 20 orbital periods. Consequently, we exclude all orbits that were integrated for less than 20 orbital periods from the frequency maps. After excluding orbits with short integration times, the frequency maps in the top row of this figure show 5704 orbits for Model A and 6168 orbits in Model B. ${ }^{11}$ The two lower panels zoom into the region of each map occupied by boxes. In both models the excluded particles were visually classified as belonging to the disk, so the frequency maps are still excellent representations of the distribution functions of the bars.

In Figure 13 the dots indicate regular orbits, while plus signs indicate chaotic orbits. The colors signify the value of an orbit's Jacobi Integral $E_{\mathrm{J}}$ (and not its initial position as in the previous maps). Particles in each map were divided into three equal groups in $E_{\mathrm{J}}$ : most negative values are colored blue, the least negative $E_{\mathrm{J}}$ values are colored red, and green indicates the intermediate range. In Model A most of the chaotic orbits are red and lie in the region labeled SPO/LPO/Disk-which marks the transition region between the disk and the bar (this is expected, e.g., from the work of Voglis et al. 2007, Contopoulos \& Harsoula 2013, and Patsis \& Katsanikas 2014b). These are the short-period orbits (SPOs) and long-

\footnotetext{
11 The deeper potential resulting from the growth of the central point mass and associated angular momentum transport causes $\sim 450$ particles to be pulled inward, and hence they execute a large number of orbital periods in the same time interval.
} 

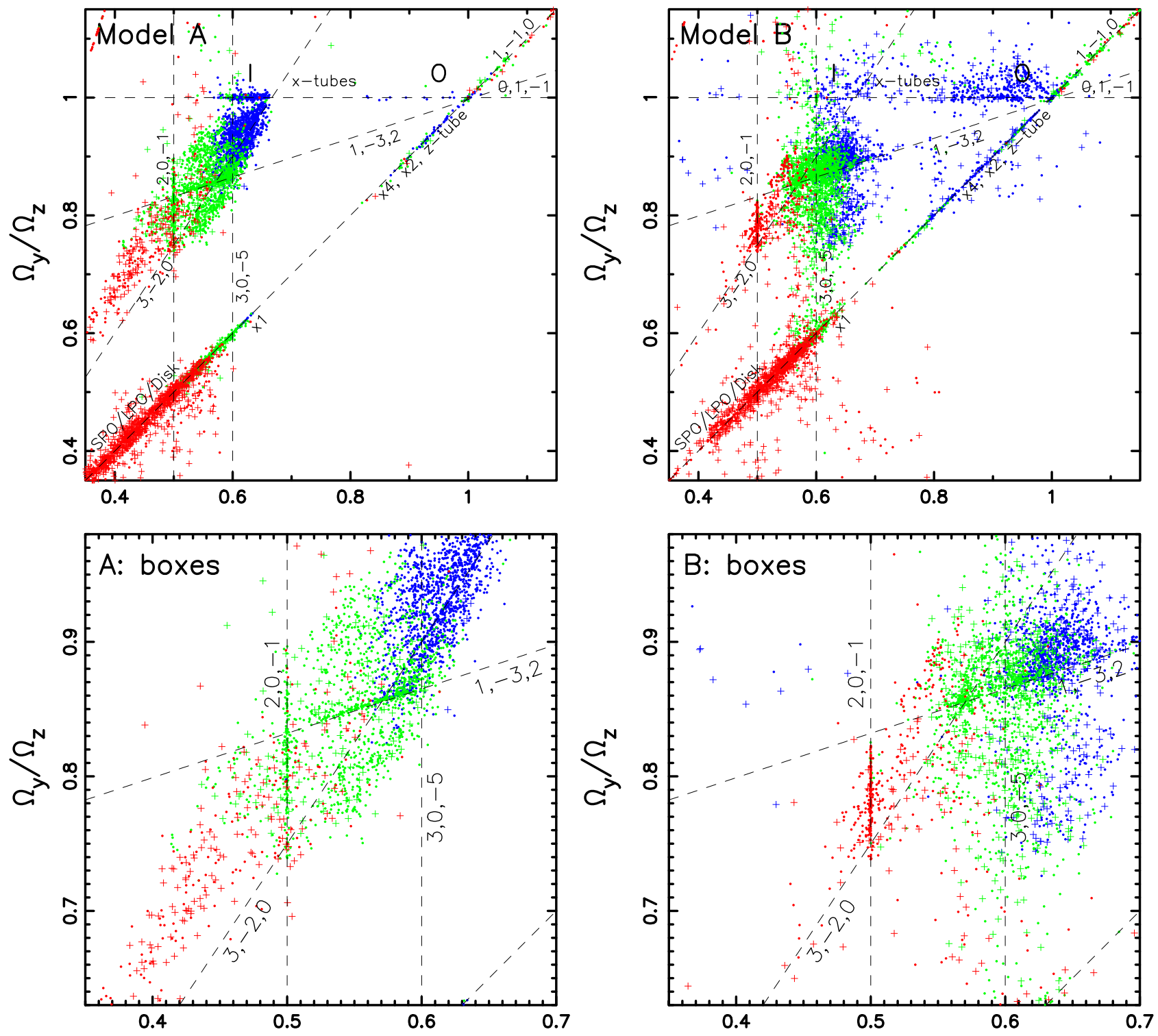

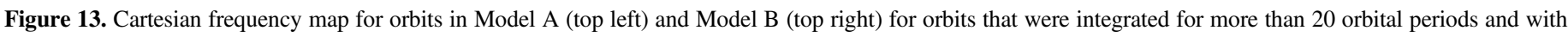

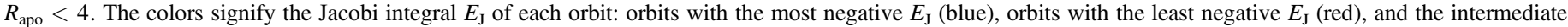

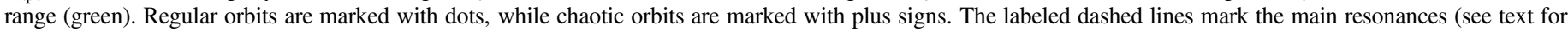
details). The lower two panels show a zoom-in of the central region of the map where box-like orbits reside.

period orbits (LPOs) that are temporarily trapped around the L4 and L5 Lagrange points. These orbits are not present in the Dehnen model (a pure ellipsoid) since they arise at the transition between the bar and disk. In Model B chaotic orbits appear in all energy ranges as a consequence of scattering by the central point mass.

In both maps points cluster along "resonances" marked by dashed lines labeled with frequency ratios. The $\mathrm{x} 1$ orbits and $z$ tube orbits parented by $\mathrm{x} 2$ and $\mathrm{x} 4$ orbits lie in the same part of the frequency map as they do in the rotating Dehnen model (Figure 12, lower panel).

A "cloud" of box orbits appears to the left of the $(1,-1,0)$ line and below the $(0,1,-1)$ line in the same part of the frequency map as box orbits in the rotating Dehnen model in Figure 12 (bottom panel). The lower two panels in Figure 13 zoom into this region to more clearly show the various resonant boxlets. The vertical line labeled " $2: 0:-1$ " marks the boxy banana orbits. The $\mathrm{x} 1$ bananas lie at the intersection between
" $2: 0:-1$ " and " $1:-1: 0$ " and are periodic orbits that satisfy the condition " $\Omega_{x}: \Omega_{y}: \Omega_{z}=2:-2:-1$ " (these are also referred to as "x1v1" orbits by Skokos et al. 2002a). Also seen is the 3: $-2: 0$ resonance. Although the "double pretzel"-like orbits (Figure 4, top row) look slightly different from the "double fish"-like orbits (Figure 4, second and third rows), they both belong to this resonance family.

The frequency map for Model B shows essentially the same features as Model A except that a number of orbits with low values of $E_{\mathrm{J}}$ (blue) are scattered around the map. These orbits were scatted by the growing central point mass and associated with inner $x$-tubes (near the label "I"), outer $x$-tubes (near label "O”), and prograde $z$-tubes.

\section{SUMMARY AND DISCUSSION}

We have analyzed 10,000 orbits from each of two selfconsistent $N$-body bar models. These 20,000 orbits were 
classified both visually and with a new automatic orbit classification method developed here. By grouping individual bar orbits into major families, we showed that each family has a counterpart in a rotating Dehnen model. This is different from the textbook view that the main body of a bar is composed primarily of prograde $\mathrm{x} 1$ orbits, prograde $\mathrm{x} 2$ orbits, and their vertical bifurcations (BT08). We examined the distributions of orbit families with radius and Jacobi integral $\left(E_{\mathrm{J}}\right)$. The phasespace distributions were examined using SoSs and frequency maps. The main results of this analysis listed below.

1. It is well known that the $\mathrm{x} 1$ orbit is the same as the $x$-axis orbit in triaxial potential subjected to high pattern speeds (Schwarzschild 1982; Martinet \& de Zeeuw 1988), and that this orbit parents the box orbit family. We demonstrate that although the $\mathrm{x} 1$ orbit is prograde, the box-like orbits parented by it have little or no net angular momentum about any axis. These box-like orbits (referred to in the bar literature as "quasi-periodic $\mathrm{x} 1$ " orbits or "fat $\mathrm{x} 1$ " orbits) dominate the distribution functions of the $N$-body bars. We also find multiple families of "resonant" boxlet orbits similar to those found in stationary triaxial potentials (see Figures 1, 3, 4).

2. $N$-body bars also contain a small fraction of long-axis tube orbits that behave exactly like those in rotating triaxial ellipsoids. These orbits are parented by the stable anomalous orbits (periodic 1:1 loops rotating about the $x$ axis), which, as predicted by Heisler et al. (1982), are tipped either clockwise or anticlockwise about the $y$-axis of the figure by the Coriolis force depending on the sign of their angular momentum $J_{x}$ (Figure 5).

3. We find no prograde $z$-tubes parented by $\mathrm{x} 2$ orbits in the pure bar model (Model A) and only a small fraction of retrograde $z$-tubes (parented by $x 4$ orbits) (Figure 6). This is consistent with the behavior of short-axis tubes found in rotating triaxial potentials - retrograde members of the family dominate at high pattern speeds (Martinet \& de Zeeuw 1988; Deibel et al. 2011). However, in Model B, which has a central point mass representing a $0.2 \%$ $\mathrm{SMBH}$, a significant fraction of the $\mathrm{x} 1$ and $x$-tube orbits are destroyed and replaced by prograde $z$-tube orbits (parented by $\mathrm{x} 2$ orbits). This behavior in response to the growth of a central point mass is consistent with previous work on the self-consistent growth of central spherical components in triaxial potentials (e.g., SS04, Valluri et al. 2010).

4. Orbit families not seen in triaxial ellipsoids only appear at the interface between the bar and the disk. These include the short-period orbits (SPOs) and long-period orbits (LPOs) that circulate around the L4 and L5 Lagrange points of the bar, as well as orbits that oscillate between the L1 and L2 Lagrange points (Athanassoula et al. 2009). There are also a large number of chaotic orbits found in this region (Harsoula \& Kalapotharakos 2009; Contopoulos \& Harsoula 2013).

5. The orbit families in bars with and without an SMBH are similar except in the central region. The growth of the SMBH causes (a) a reduction in the fraction of $\mathrm{x} 1$ orbits and $x$-tubes; (b) an increase in the fraction of $z$-tubes, especially the prograde variety, which were previously absent; (c) an increase in the fraction of chaotic orbits (Figure 8); and (d) a new population of PKOs. All these effects arise because the SMBH scatters orbits with small pericenter radii and enhances mass inflow due to adiabatic contraction, aided by angular momentum transport by the bar.

6. The three new families of PKOs are found near the SMBH: here the Keplerian potential of the SMBH dominates, but orbits experience precession owing to the potential of the rotating triaxial bar. Interestingly, when integrated for long times, the orbits in this region are found to belong to the same three major orbit families: boxes, long-axis tubes, and short-axis tubes that are found in the main body of the bar. About $2 \%$ of bar orbits (113/ 6168) were found to be PKOs: a total mass fraction comparable to that of the SMBH itself. This family also has counterparts in stationary triaxial potentials with SMBHs that are called "saucers" (z-tubes) and "pyramids" (boxes) (Sridhar \& Touma 1999; Poon \& Merritt 2001; Merritt \& Vasiliev 2011; Li et al. 2015).

7. A new automated orbit classification scheme for bar orbits based on Cartesian fundamental frequencies largely recovers the same classifications as those obtained by visually examining 20,000 individual orbits. A comparison of frequency maps of orbits in a rotating Dehnen model and orbits drawn from self-consistent bars provides further confirmation that the two systems are fundamentally similar.

Our effort to unify the orbital structure of bars and triaxial ellipsoids goes beyond mere theoretical curiosity. Recent developments make this new unified picture important for observational applications. Recently, we studied simulations of barred galaxies with SMBHs (Brown et al. 2013; Hartmann et al. 2014) to show that a bar can cause an increase in the central line-of-sight velocity dispersion $(\sigma)$ by about $7 \%-25 \%$ -an increase that is consistent with the average offset observed for barred galaxies relative to unbarred ones from the $M_{\mathrm{BH}}-\sigma$ relation. In addition, a more serious consequence of the presence of a bar is that its unique orbital structure (the combination of the radially biased bar orbits and the high bar pattern speed) has been shown to result in a high central velocity dispersion but negative fourth Gauss-Hermite parameters $\left(h_{4}\right)$, even in the vicinity of the black hole. The Schwarzschild orbit superposition method is currently a popular way to measure SMBH masses from stellar kinematics. Brown et al. (2013) showed that this unique combination of kinematical parameters (high central $\sigma$ and negative $h_{4}$ ) can result in a systematic overestimate of the mass of the SMBH if the bar is modeled as if it is axisymmetric and the true nature of bar orbits is not taken into consideration. The axisymmetric and stationary triaxial Schwarzschild modeling methods are currently considered the "gold standards" for dynamical black hole mass determination against which secondary methods (e.g., reverberation mapping) are calibrated. However, making incorrect assumptions about the form of the potential can bias the best-fit black hole mass. For instance, it was shown by van den Bosch \& de Zeeuw (2010) that if a nearly axisymmetric galaxy was modeled by a stationary triaxial code, the best-fit black hole mass nearly doubled. A bias in $M_{\mathrm{BH}}$ (which can be a factor of two or higher depending on the orientation of the bar) was dramatically illustrated in the case of the barred Seyfert 1 galaxy NGC 4151 (Onken et al. 2014). In NGC 4151 although the central bulge appears very circular, there is clear kinematical evidence for a bar seen in the velocity fields, e.g., clear isophotal twists in line-of-sight velocity and negative $h_{4}$ 
parameters along the length of the bar axis. Although nearly $60 \%$ of spiral/S0 galaxies with existing stellar dynamical black hole mass measurements are in barred galaxies, they have been derived with axisymmetric models!

Since the Schwarzschild method relies on the superposition of orbits, the results are extremely sensitive to the completeness and accuracy of the library of orbits that is supplied to the superposition code (Thomas et al. 2004; Valluri et al. 2004). Currently, there are no orbit superposition codes designed to measure black hole masses from stellar kinematical data in bars. As the work in this paper demonstrates, the textbook view that the orbits in a bar arise from perturbations to a series of prograde $\mathrm{x} 1$ and $\mathrm{x} 2$ orbits is incomplete since it ignores nonrotating boxes, retrograde $z$-tubes (parented by $\mathrm{x} 4$ orbits), and long-axis tubes. In fact, as shown in Figure 11, prograde $z$ tubes (parented by $\mathrm{x} 2$ orbits) are only produced by the action of an SMBH (they may also be produced by dissipative gas inflow as shown by Debattista et al. 2015).

There is a substantial body of literature on Schwarzschild modeling of triaxial ellipsoids with and without figure rotation (e.g., Schwarzschild 1979, 1982; van den Bosch et al. 2008; Vasiliev 2013), which has recently been extended to modeling bars (Wang et al. 2013; Vasiliev \& Athanassoula 2015). A new unified framework for understanding the orbital structure of bars, especially bars with self-consistently grown SMBHs, will make it possible to construct more realistic Schwarzschild models for barred disk galaxies. This is important for ensuring that black hole masses at the low end of the $M_{\mathrm{BH}}-\sigma$ relation are accurately measured, since systematic overestimates of black hole masses in barred disks would erase morphological differences between the black hole scaling relations of disks and ellipticals, which could be crucial to understanding the coevolution of black holes and their host galaxies.

The authors wish to thank P. T. de Zeeuw for helpful comments and an anonymous referee for a constructive report - both of which improved the presentation of this paper. M.V. and C.A. were supported in part by University of Michigan's Office of Research, HST-AR-13890.001, NSF award AST0908346, and NASA-ATP award NNX15AK79G. J.S. is partially supported by the 973 Program of China under grant no. 2014CB845700, by the National Natural Science Foundation of China under grant nos.11333003, 11322326, and 11073037 , and by the Strategic Priority Research Program "The Emergence of Cosmological Structures" (no. XDB09000000) of the Chinese Academy of Sciences. This work made use of the facilities of the Center for High Performance Computing at Shanghai Astronomical Observatory. V.P.D. is supported by STFC Consolidated grant no. ST/ M000877/1 and partially supported by the Chinese Academy of Sciences President's International Fellowship Initiative (PIFI, Grant no. 2015VMB004).

\section{APPENDIX A \\ ORBITAL FREQUENCY ANALYSIS}

In this section we provide a brief description of the orbital frequency analysis method first introduced by Binney \& Spergel (1982, 1984) and further developed by Laskar (1990, 1993). In Hamiltonian dynamics the angle variables and their canonically conjugate actions $J_{i}$ uniquely define a regular orbit (BT08). The time derivatives of three angle variables are the fundamental frequencies $\dot{\theta}_{i}(t)=\Omega_{i}$. Orbits in galaxies are approximately quasi-periodic (BT08); hence, their space and velocity coordinates can be represented by time series of the form

$$
x(t)=\sum_{k=1}^{k_{\max }} A_{k} e^{i \omega_{k} t},
$$

with similar expressions for $y(t), z(t)$, and velocity components, $V_{x}(t), V_{y}(t), V_{z}(t)$. The amplitudes $A_{k}$ and the frequencies $\omega_{k}$ can be obtained by taking a Fourier transform (with a window function) of a time series $f(t)$ constructed from the spatial or/and velocity coordinates of an orbit. For a regular orbit in a three-dimensional potential, only three frequencies in the spectrum, $\Omega_{i}, i=1, \ldots, 3$, are linearly independent, and all other frequencies in the spectrum can be written as integer linear combinations of these three frequencies; therefore, the $\Omega_{i}, i=1, \ldots, 3$ are referred to as "fundamental frequencies." Laskar (1990, 1993) and Papaphilippou \& Laskar (1996, 1998) developed an accurate numerical technique "Numerical Analysis of Fundamental Frequencies" to recover frequencies in completely general potentials. In this paper we use the implementation of this algorithm ${ }^{12}$ first presented in Valluri \& Merritt (1998) and subsequently modified to work with orbits in $N$-body potentials (Valluri et al. 2010). With this code the frequency components in the spectrum can be recovered with high accuracy ( 1 part in $10^{-5}$ or better) in $\sim 20-30$ orbital periods. Our code uses integer programming to recover orbital fundamental frequencies. We have applied this frequency analysis method to orbits of particles in simulations of triaxial halos (Valluri et al. 2010, 2012), to the orbits of halo stars and dark matter particles in fully cosmological-hydrodynamical simulations (Valluri et al. 2013), and to orbits in rotating triaxial potentials (Deibel et al. 2011).

To determine the fraction of chaotic orbits in a potential, the orbital time series is divided into two equal segments and the orbital fundamental frequencies are computed in each time segment. Since regular orbits have fixed frequencies that do not change with time, the change in the frequency measured in the two time segments can be used to measure the drift in frequency space. The "frequency drift" for each frequency component $\Omega_{i}$ can be computed as

$$
\log \left(\Delta f_{i}\right)=\log \left|\frac{\Omega_{i}\left(t_{1}\right)-\Omega_{i}\left(t_{2}\right)}{\Omega_{i}\left(t_{1}\right)}\right| .
$$

We define the frequency drift parameter $\log (\Delta f)$ (logarithm to base 10) for an orbit to be the value associated with the fundamental frequency $\Omega_{i}$ with the largest amplitude in the Fourier spectrum. The larger the value of the frequency drift parameter, the more chaotic the orbit. Since the frequency difference is normalized by the absolute value of the frequency, it is possible to compare diffusion rates of orbits with a wide range of orbital periods. Valluri et al. (2010) showed that even for orbits in frozen $N$-body potentials (where most orbits are affected by discreetness noise), it is possible to distinguish between $N$-body jitter and true chaos. In their tests orbits with $\log (\Delta f)>-1.2$ were defined as chaotic. We use the same criterion in this paper.

\footnotetext{
${ }^{12}$ Made available publicly at http://dept.astro.lsa.umich.edu/ mvalluri/ resources.html.
} 

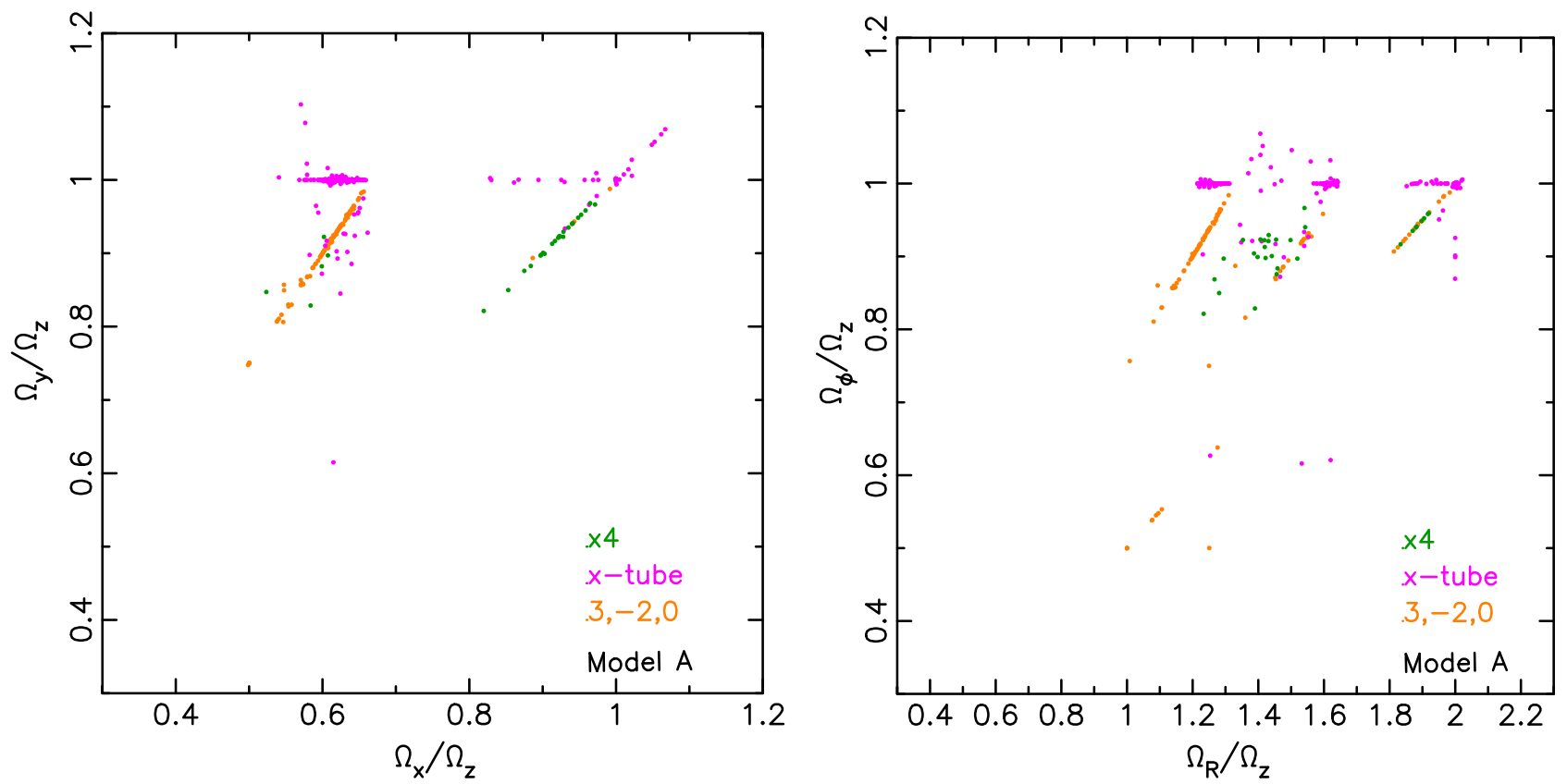

Figure 14. Frequency maps constructed from fundamental frequencies in Cartesian coordinates (left) and cylindrical polar coordinates (right). Only three orbit families from Model A, color-coded on the basis of their visual classification, are plotted: $x$-tubes (pink dots), retrograde $z$-tubes parented by $\mathrm{x} 4$ orbits (green dots), and 3:-2:0 resonant orbits (orange dots). Resonance lines are not marked, but orbits can be seen to clearly lie along three main resonances (each associated with one primary orbit family) in the Cartesian map (left). In the cylindrical frequency map the same families are split into multiple groups—many of which overlap-making it difficult to use these frequencies to classify orbits.

\section{APPENDIX B \\ AUTOMATED BAR ORBIT CLASSIFICATION}

Automated classification of orbits in triaxial $N$-body models is based on orbital fundamental frequencies. The method is well developed and has been utilized in the past (Carpintero \& Aguilar 1998; Valluri et al. 2010). We refer the reader to these papers for further details. In this appendix we describe a similar automatic classification scheme for orbits in bars.

Voglis et al. (2007) used orbital fundamental frequencies in cylindrical polar coordinates in the frame co-rotating with the bar $\left(\Omega_{R}, \Omega_{\phi}, \Omega_{z}\right)$ to make frequency maps and characterize regular and chaotic orbits in their $N$-body bars. However, as we now discuss, we find that orbital frequencies in Cartesian coordinates provide a more robust means for bar orbit classification.

Figure 14 shows frequency maps constructed from fundamental frequencies computed in Cartesian coordinates in the frame co-rotating with the bar (left) and in cylindrical polar coordinates (right). Three orbit families, selected on the basis of visual classification of orbits in Model, A are plotted: $x$-tubes (pink dots), retrograde $z$-tubes parented by $\mathrm{x} 4$ orbits (green dots), and 3:-2:0 resonant orbits (orange dots). In the map on the left, the three families lie in reasonably (thought not perfectly) separate regions of the map. The separation of the different orbit families seen in a Cartesian frequency map (also in Figure 13) is quite similar to the separation found in self-consistent stationary triaxial potentials (Valluri et al. 2012).

In the cylindrical frequency map (Figure 14, right panel) each orbit type (i.e., points of a single color) appears in multiple groups and there is significant overlap between the different types. We do not plot the box orbits in these plots (the most numerous population) to avoid overcrowding the maps. It can be seen in Figure 13 that the box orbits are concentrated in the cloud of points to the left of the diagonal. However, in cylindrical coordinates this family is split into multiple groups that overlap with other families. Voglis et al. (2007) used frequency maps in cylindrical coordinates in which box-like orbits were separated into multiple groups. They used these groupings to classify orbits into $\mathrm{x} 1$ and $\mathrm{x} 4$ orbits, several resonant families, and two large groups that they called "Group A" and "Group B." Our maps in cylindrical coordinates also showed similar groups (e.g., Figure 14, right panel), but we found that the different groups were not characterized by unique qualitative or quantitative properties.

Since our primary objective is to use orbital frequencies to automatically classify orbits, the larger degree of separation of different orbit families (boxes, $x$-tubes, $z$-tubes, and $\mathrm{x} 1$ orbits) in Cartesian frequency maps, though not perfect, in combination with orbital angular momentum and orbital elongation, results in the robust orbit classification scheme described below.

Our orbit classification algorithm begins by identifying the location of the end of the bar. In Figure 15 the black and red histograms shows the apocenter radii of all orbits in each of the two models. The red histograms show the distribution of apocenter radii of all orbits that were visually classified as "disk" orbits (including the short/long-period orbits). Both histograms show a clear break in $R_{\text {apo }}$. This break coincides with the apocenter radius beyond which the majority of the visually classified disk orbits lie; consequently, $R_{\text {apo }}=4$ defines the transition between the bar and the disk. The few disk orbits with $R_{\text {apo }}<$ the break radius are classified as $z$-tubes by the automated classifier. All orbits that lie beyond the bar are automatically classified as "disk" orbits.

For each orbit the three fundamental frequencies in Cartesian coordinates $\Omega_{x}, \Omega_{y}, \Omega_{z}$ are obtained using frequency analysis. 

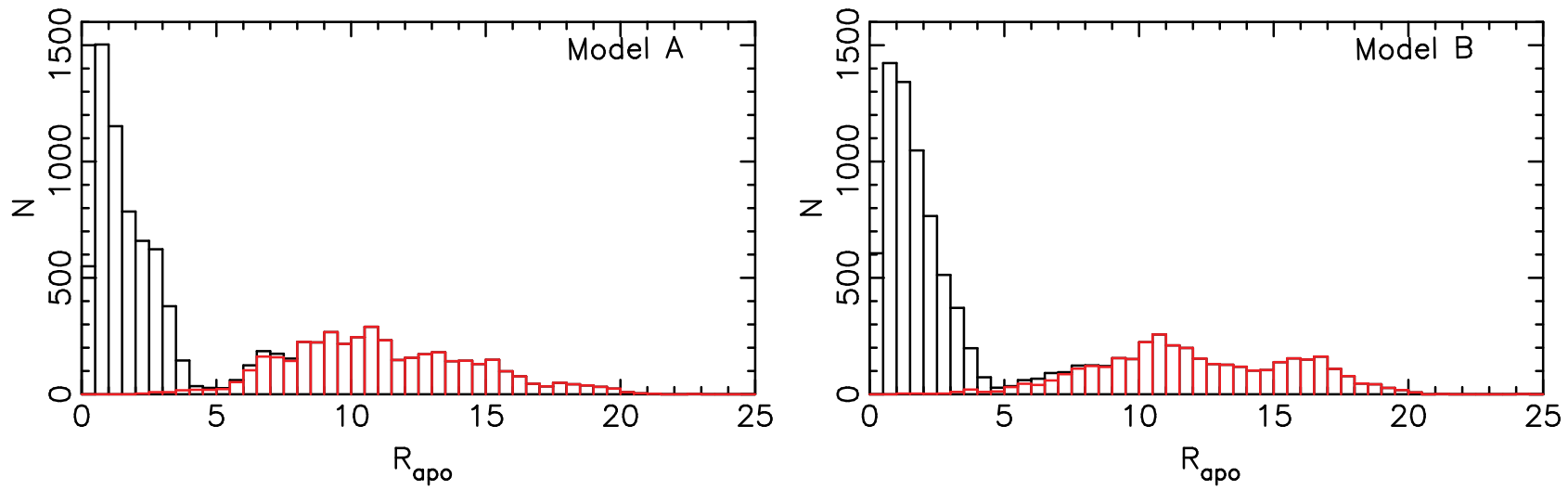

Figure 15. Histograms of apocenter radii of 10,000 orbits in Model A (left) and Model B (right). The black histograms shows apocenter radii of all 10,000 orbits in the model (determined by integrating each orbit for $t=1000$ in program units). The red histograms show the distribution of apocenter radii of all orbits that were visually classified as disk orbits. The clear break at $\sim 4$ in both models coincides with the value of $r_{\text {apo }}$ beyond which the majority of the disk orbits are found and is used to define the end of the bar.

We then determined which (if any) of the frequency ratios were rational, i.e., if any pair of frequencies $\Omega_{i} / \Omega_{j}=n_{i} / n_{j}$, where $n_{i}, n_{j}, n_{k}<10$ ( $i, j, k$ corresponding to $x, y, z$ coordinates, respectively). For determining whether the frequency ratios are rational, we multiply both the denominator and numerator by successive integers $(<10)$ and compute the difference between the new numerator (denominator) and the integer, until this difference is less than 0.01 . This condition allows us to identify both strictly periodic or resonant orbits and those that are associated with a periodic or resonant family but are not strictly periodic (closed).

If all three frequency ratios are rational, i.e., if $\Omega_{x} / \Omega_{y}=n_{i} / n_{j}, \quad \Omega_{y} / \Omega_{z}=n_{j} / n_{k}$ and hence $\Omega_{z} / \Omega_{x}=n_{k} / n_{i}$ and $n_{i} \neq n_{j} \neq n_{k}$, the orbit is a closed (periodic) box orbit. If $n_{j}=n_{k} \neq n_{i}$, the orbit is a closed periodic long-axis tube orbit. However, if $n_{i}=n_{j} \neq n_{k}$, the orbit is a closed periodic $\mathrm{x} 1$ orbit. (Note that if two pairs of frequency ratios are rational, then the third pair must be rational as well.)

Only one pair of frequency ratios is rational, e.g., if $\Omega_{y} / \Omega_{z}=n_{j} / n_{k}$, then if $n_{j}=n_{k}$, the orbit loops around the short axis ( $z$ tube, $\mathrm{x} 2, \mathrm{x} 4)$, but if $n_{j} \neq n_{k}$, the orbit is an open (resonant) boxlet. Likewise, if $\Omega_{y} / \Omega_{z}=n_{j} / n_{k}$ and if $n_{j}=n_{k}$, then the orbit is an open long-axis tube, but if $n_{j} \neq n_{k}$, the orbit is also an open boxlet.

These conditions are essentially identical to those used to classify orbits in triaxial potentials into boxes, $z$-tubes, and $x$ tubes (Carpintero \& Aguilar 1998; Valluri et al. 2010). In addition to these conditions on orbital frequencies, for some types of orbits we required that certain conditions on the time-averaged normalized angular momenta be satisfied. For retrograde $z$-tubes $\left\langle J_{z}\right\rangle<-0.95$ and for prograde $z$-tubes $\left\langle J_{z}\right\rangle>+0.95$.

Classical x1 orbits are those for which $\Omega_{x} / \Omega_{y} \sim 1$ and $\Omega_{y} / \Omega_{z}<0.7$ (determined empirically from the frequency map). We also find that box orbits (as classified above) that have $\left\langle J_{z}\right\rangle>+0.6$ and $|y|_{\max } /|x|_{\max }<0.35$ (see Figure 2) are classical $\mathrm{x} 1$ orbits. We therefore use these two criteria to reclassify orbits that may have been at first classified as boxes or $\mathrm{x} 1$ orbits. Banana orbits are boxes or $\mathrm{x} 1$ orbits that also satisfy the condition $\Omega_{z}: \Omega_{x}=2: 1$. Brezel orbits are boxes that also satisfy the condition $\Omega_{z}: \Omega_{x}=-5: 3$. The "3:-2:0" resonant orbits satisfy the condition $\Omega_{x} / \Omega_{y}=3:-2$.

\section{REFERENCES}

Adams, F. C., Bloch, A. M., Butler, S. C., Druce, J. M., \& Ketchum, J. A. 2007, ApJ, 670, 1027

Aguerri, J. A. L., Debattista, V. P., \& Corsini, E. M. 2003, MNRAS, 338, 465

Aguerri, J. A. L., Méndez-Abreu, J., Falcón-Barroso, J., et al. 2015, A\&A, 576, A102

Arnold, V. I. 1978, Mathematical Methods of Classical Mechanics (Berlin: Springer)

Athanassoula, E. 1992, MNRAS, 259, 345

Athanassoula, E. 2005, MNRAS, 358, 1477

Athanassoula, E., \& Misiriotis, A. 2002, MNRAS, 330, 35

Athanassoula, E., Romero-Gómez, M., \& Masdemont, J. J. 2009, MNRAS, 394, 67

Athanassoula, E., \& Sellwood, J. A. 1986, MNRAS, 221, 213

Binney, J., \& Spergel, D. 1982, ApJ, 252, 308

Binney, J., \& Spergel, D. 1984, MNRAS, 206, 159

Binney, J., \& Tremaine, S. 2008, Galactic Dynamics (2nd ed.; Princeton, NJ: Princeton Univ. Press)

Brown, J. S., Valluri, M., Shen, J., \& Debattista, V. P. 2013, ApJ, 778, 151

Carpintero, D. D., \& Aguilar, L. A. 1998, MNRAS, 298, 1

Combes, F., \& Sanders, R. H. 1981, A\&A, 96, 164

Contopoulos, G., \& Grosbol, P. 1989, A\&ARv, 1, 261

Contopoulos, G., \& Harsoula, M. 2013, MNRAS, 436, 1201

Contopoulos, G., \& Papayannopoulos, T. 1980, A\&A, 92, 33

Corsini, E. M. 2011, MSAIS, 18, 23

Debattista, V. P., Corsini, E. M., \& Aguerri, J. A. L. 2002, MNRAS, 332, 65

Debattista, V. P., Ness, M., Earp, S. W. F., \& Cole, D. R. 2015, ApJL, 812 L16

Debattista, V. P., \& Williams, T. B. 2004, ApJ, 605, 714

Dehnen, W. 1993, MNRAS, 265, 250

Deibel, A. T., Valluri, M., \& Merritt, D. 2011, ApJ, 728, 128

de Zeeuw, T. 1985a, MNRAS, 216, 273

de Zeeuw, T. 1985b, MNRAS, 215, 731

de Zeeuw, T., \& Merritt, D. 1983, ApJ, 267, 571

Gebhardt, K., Richstone, D., Ajhar, E. A., et al. 1996, AJ, 112, 105

Harsoula, M., \& Kalapotharakos, C. 2009, MNRAS, 394, 1605

Hartmann, M., Debattista, V. P., Cole, D. R., et al. 2014, MNRAS, 441, 1243

Heiligman, G., \& Schwarzschild, M. 1979, ApJ, 233, 872

Heisler, J., Merritt, D., \& Schwarzschild, M. 1982, ApJ, 258, 490

Hemsendorf, M., \& Merritt, D. 2002, ApJ, 580, 606

Laskar, J. 1990, Icar, 88, 266

Laskar, J. 1993, CeMDA, 56, 191

Lauer, T. R., Gebhardt, K., Faber, S. M., et al. 2007, ApJ, 664, 226

Li, B., Holley-Bockelmann, K., \& Khan, F. 2015, ApJ, 811, 25

Martinet, L., \& de Zeeuw, T. 1988, A\&A, 206, 269

Meidt, S. E., Rand, R. J., Merrifield, M. R., Debattista, V. P., \& Shen, J. 2008, ApJ, 676, 899

Merrifield, M. R., \& Kuijken, K. 1995, MNRAS, 274, 933

Merritt, D., \& Fridman, T. 1996, ApJ, 460, 136

Merritt, D., \& Valluri, M. 1999, AJ, 118, 1177

Merritt, D., \& Vasiliev, E. 2011, ApJ, 726, 61

Miller, R. H. 1964, ApJ, 140, 250 
Miralda-Escude, J., \& Schwarzschild, M. 1989, ApJ, 339, 752

Onken, C. A., Valluri, M., Brown, J. S., et al. 2014, ApJ, 791, 37

Papaphilippou, Y., \& Laskar, J. 1996, A\&A, 307, 427

Papaphilippou, Y., \& Laskar, J. 1998, A\&A, 329, 451

Patsis, P. A., Kalapotharakos, C., \& Grosbøl, P. 2010, MNRAS, 408, 22

Patsis, P. A., \& Katsanikas, M. 2014a, MNRAS, 445, 3525

Patsis, P. A., \& Katsanikas, M. 2014b, MNRAS, 445, 3546

Patsis, P. A., Skokos, C., \& Athanassoula, E. 2002, MNRAS, 337, 578

Patsis, P. A., Skokos, C., \& Athanassoula, E. 2003, MNRAS, 342, 69

Patsis, P. A., \& Zachilas, L. 1990, A\&A, 227, 37

Pfenniger, D. 1984, A\&A, 134, 373

Pfenniger, D., \& Friedli, D. 1991, A\&A, 252, 75

Poon, M. Y., \& Merritt, D. 2001, ApJ, 549, 192

Portail, M., Wegg, C., \& Gerhard, O. 2015, MNRAS, 450, L66

Raha, N., Sellwood, J. A., James, R. A., \& Kahn, F. D. 1991, Natur, 352, 411

Schwarzschild, M. 1979, ApJ, 232, 236

Schwarzschild, M. 1982, ApJ, 263, 599

Schwarzschild, M. 1993, ApJ, 409, 563

Sellwood, J. A. 2014a, arXiv:1406.6606

Sellwood, J. A. 2014b, RvMP, 86, 1

Sellwood, J. A., \& Valluri, M. 1997, MNRAS, 287, 124

Sellwood, J. A., \& Wilkinson, A. 1993, RPPh, 56, 173

Shen, J., \& Sellwood, J. A. 2004, ApJ, 604, 614 (SS04)

Skokos, C., Patsis, P. A., \& Athanassoula, E. 2002a, MNRAS, 333, 847
Skokos, C., Patsis, P. A., \& Athanassoula, E. 2002b, MNRAS, 333, 861

Sparke, L. S., \& Sellwood, J. A. 1987, MNRAS, 225, 653

Sridhar, S., \& Touma, J. 1999, MNRAS, 303, 483

Statler, T. S. 1987, ApJ, 321, 113

Thomas, J., Saglia, R. P., Bender, R., et al. 2004, MNRAS, 353, 391

Toomre, A. 1966, Geophysical Fluid Dynamics (Woods Hole, MA: Oceanographic Inst.)

Tremaine, S., Richstone, D. O., Byun, Y., et al. 1994, AJ, 107, 634

Tremaine, S., \& Weinberg, M. D. 1984, ApJL, 282, L5

Valluri, M., Debattista, V. P., Quinn, T., \& Moore, B. 2010, MNRAS, 403, 525

Valluri, M., Debattista, V. P., Quinn, T. R., Roškar, R., \& Wadsley, J. 2012, MNRAS, 419, 1951

Valluri, M., Debattista, V. P., Stinson, G. S., et al. 2013, ApJ, 767, 93

Valluri, M., \& Merritt, D. 1998, ApJ, 506, 686

Valluri, M., Merritt, D., \& Emsellem, E. 2004, ApJ, 602, 66

van Albada, T. S., Kotanyi, C. G., \& Schwarzschild, M. 1982, MNRAS, 198, 303

van den Bosch, R. C. E., \& de Zeeuw, P. T. 2010, MNRAS, 401, 1770

van den Bosch, R. C. E., van de Ven, G., Verolme, E. K., Cappellari, M., \& de Zeeuw, P. T. 2008, MNRAS, 385, 647

Vasiliev, E. 2013, MNRAS, 434, 3174

Vasiliev, E., \& Athanassoula, E. 2015, MNRAS, 450, 2842

Voglis, N., Harsoula, M., \& Contopoulos, G. 2007, MNRAS, 381, 757

Wang, Y., Mao, S., Long, R. J., \& Shen, J. 2013, MNRAS, 435, 3437 